\title{
CITED2 functions as a molecular switch of cytokine-induced proliferation and quiescence
}

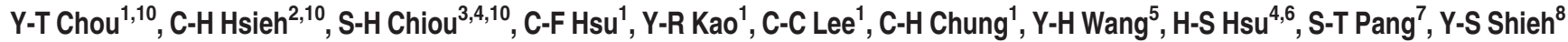 \\ and $\mathrm{C}-\mathrm{W} \mathrm{Wu}^{*, 1,2,4,9}$
}

Transforming growth factor- $\alpha$ (TGF- $\alpha$ )-induced proliferation and transforming growth factor- $\beta$ (TGF- $\beta$ )-mediated quiescence are intricately balanced in normal lung-tissue homeostasis but are deregulated during neoplastic progression of lung cancer. Here, we show that $\mathrm{Cbp} / \mathrm{p} 300$-interacting transactivator with Glu/Asp-rich carboxy-terminal domain 2 (CITED2), a novel MYCinteracting transcriptional modulator, responds to TGF- $\alpha$ induction and TGF- $\beta$ suppression to orchestrate cellular proliferation and quiescence, respectively. Upon TGF- $\alpha$ induction, CITED2 was induced by MYC and further modulated MYC-mediated transcription in a feed-forward manner. CITED2 recruited p300 to promote MYC-p300-mediated transactivation of E2F3, leading to increased G1/S cell cycle progression. Moreover, CITED2 inhibited cellular quiescence by enhancing MYC-mediated suppression of $p 21^{C I P 1}$. CITED2 interacted with histone deacetylase 1 (HDAC1) and potentiated MYC-HDAC1 complex formation. TGF- $\beta$ stimulation provoked downregulation of CITED2, which abrogated MYC-HDAC1-mediated p21 ${ }^{C I P 1}$ suppression, causing cellular quiescence. Ectopic CITED2 expression enhanced tumor growth in nude mice; furthermore, CITED2 knockdown caused tumor shrinkage and increased overall host mouse survival rates. Expression of CITED2/MYC/E2F3/ p21 ${ }^{\mathrm{CIP} 1}$ signaling molecules was associated with poor prognosis of lung cancer patients. Thus, CITED2 functions as a molecular switch of TGF- $\alpha$ and TGF- $\beta$-induced growth control, and MYC-CITED2 signaling axis provides a new index for predicting clinical outcome.

Cell Death and Differentiation (2012) 19, 2015-2028; doi:10.1038/cdd.2012.91; published online 20 July 2012

Transforming growth factor (TGF)- $\alpha$ and transforming growth factor (TGF)- $\beta$ are two pivotal cytokines that regulate proliferation and quiescence, respectively, of lung epithelial cells during both normal and neoplastic lung development. ${ }^{1}$ Epidermal growth factor receptor (EGFR) is often amplified or mutated in non-small-cell lung cancer (NSCLC), ${ }^{2}$ and TGF- $\alpha$, one of the EGFR cognate ligands, is frequently overexpressed and causes receptor hyperactivity in autocrine loops in cancer cells isolated from patients with NSCLC. ${ }^{3}$ In contrast, TGF- $\beta$ induces quiescence of lung epithelial cells. ${ }^{4}$ TGF- $\beta$ signaling is mediated through the TGF- $\beta$ type II (TGFBRII) and type I (TGFBRI) receptors; methylation silencing of TGFBRII is observed in some NSCLC cancer cells, ${ }^{5}$ rendering tumor cells refractory to TGF- $\beta$-induced growth arrest. ${ }^{6}$

Cbp/p300-interacting transactivator with Glu/Asp-rich carboxy-terminal domain 2 (CITED2), which was originally identified from the studies of differential gene expression by cytokine stimulation, encodes a transcriptional modulator without DNA-binding domains. ${ }^{7}$ Mice without CITED2 display abnormal fetal lung development, including reduced terminal sacs and aberrant differentiation of alveolar epithelial cells. ${ }^{8}$ CITED2 is induced by cytokines such as interleukin-1 $\alpha,-2,-4$, -6 , -9 and -11 ; granulocyte/macrophage colony-stimulating factor; interferon- $\gamma$; platelet-derived growth factor and insulin. ${ }^{7}$ In contrast, CITED2 is inhibited by TGF- $\beta$ through a posttranscriptional control mechanism. ${ }^{9}$ CITED2 interacts with p300/CBP, Lhx2, TFAP2, Smad2/Smad3 and PPAR $\gamma$, thereby modulating transcription mediated by these transcription factors. ${ }^{10-14}$

$M Y C$, a potent oncogene, is highly expressed in bronchial epithelium basal cells and hyperplastic alveolar type II pneumocytes. ${ }^{15}$ Through the regulation of E2F transcription factor expression, MYC has a critical role in cell cycle control. ${ }^{16,17}$ Among the E2Fs, E2F3 is overexpressed in most lung cancers ${ }^{18}$ and is induced by EGFR to transactivate genes involved in the G1/S transition. ${ }^{19,20}$ In addition, MYC binds to the $p 21^{C I P 1}$ promoter, causing

\footnotetext{
${ }^{1}$ Institute of Biomedical Sciences, Academia Sinica, Taipei, ROC; ${ }^{2}$ Institute of Microbiology and Immunology, National Yang-Ming University, Taipei, ROC; ${ }^{3}$ Institute of Pharmacology, National Yang-Ming University, Taipei, ROC; ${ }^{4}$ Institute of Clinical Medicine, National Yang-Ming University, Taipei, ROC; ${ }^{5}$ Division of General Surgery, Department of Urology, Shung Ho Hospital, Taipei, ROC; ${ }^{6}$ Department of Surgery, Taipei Veterans General Hospital, Taipei, ROC; ${ }^{7}$ Division of Urology, Department of Surgery, College of Medicine, Chang Gung Memorial Hospital and University, Taoyuan, ROC; ${ }^{8}$ Department of Oral Diagnosis \& Pathology, Tri-Service General Hospital, Taipei, ROC and ${ }^{9}$ Institute of Biochemistry and Molecular Biology, National Yang-Ming University, Taipei, ROC

${ }^{*}$ Corresponding author: C-W Wu, Institute of Microbiology and Immunology, National Yang-Ming University, No.155, Sec. 2, Linong Street, Taipei 112, ROC.

Tel: +886 22826 7919; Fax: +886 22823 6518; E-mail: cwwu@ym.edu.tw

${ }^{10}$ These authors contributed equally to this work.

Keywords: CITED2; MYC; cytokine; transcriptional modulator; lung cancer

Abbreviations: CITED2, Cbp/p300-interacting transactivator with Glu/Asp-rich carboxy-terminal domain 2; TGF- $\alpha$, transforming growth factor alpha; TGF- $\beta$, transforming growth factor beta; NSCLC, non-small-cell lung cancer; EGFR, epidermal growth factor receptor; TGFBRI, transforming growth factor beta receptor I; TGFBRII, transforming growth factor beta receptor II; p300, E1A binding protein p300; HDAC1, histone deacetylase 1; Dox, doxycycline; eGFP, enhanced green fluorescent protein; shRNA, short hairpin RNA; GST, glutathione S-transferase

Received 10.4.12; revised 06.6.12; accepted 14.6.12; Edited by BD Dynlacht; published online 20.7.12
} 
transcriptional suppression of $p 21^{C I P 1} .^{21,22}$ TGF- $\beta$ induces quiescence of epithelial cells through suppression of $M Y C$ and activation of the $p 21^{C I P 1}$-mediated senescence pathway. ${ }^{23}$
Although it is known that MYC mediates abnormal cytokine signaling to induce tumorigenesis, how MYC can respond to signals from diverse cytokines and function as both a transcriptional activator and repressor to regulate cell cycle a

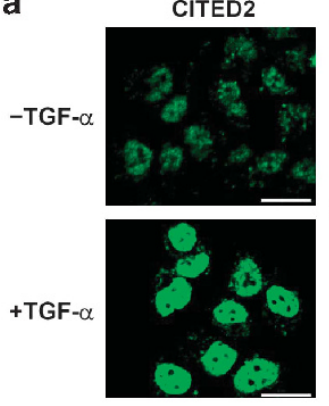

b

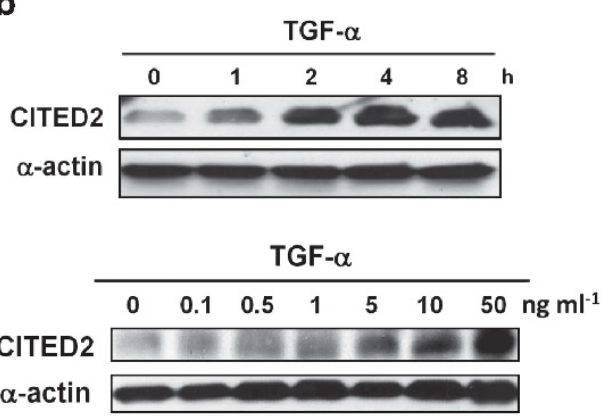

DAPI
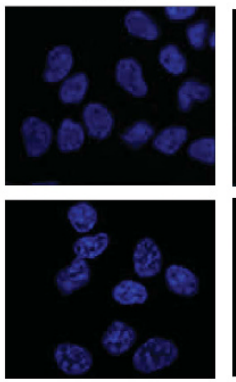

Merge

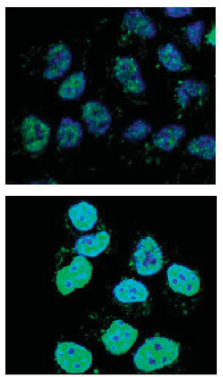

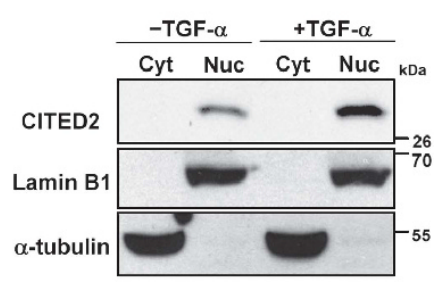

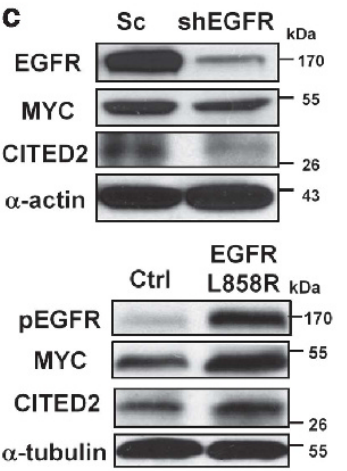

d

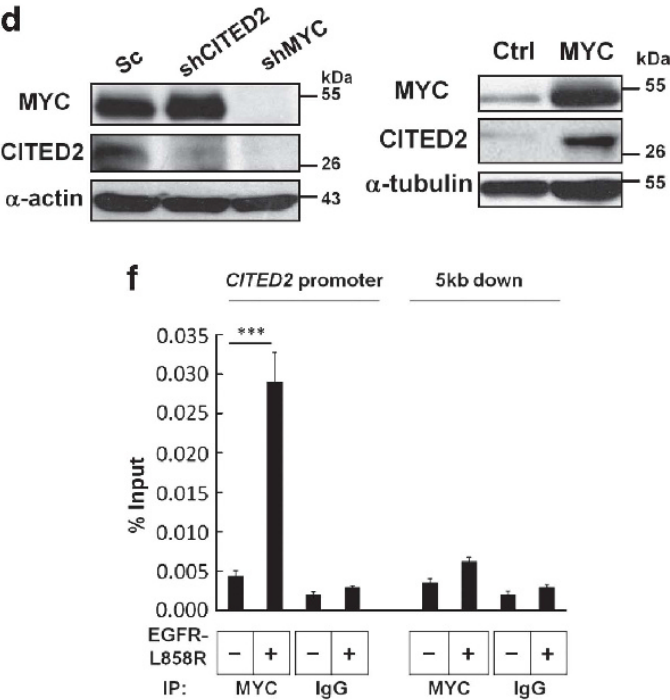

e

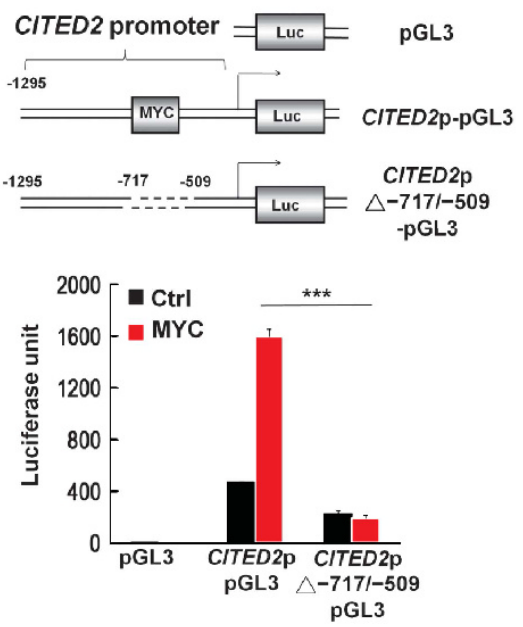

Figure 1 CITED2 is induced by TGF- $\alpha$ /EGFR signaling. (a) Representative image of confocal immunofluorescence staining of CITED2 expression in A549 cells treated with or without TGF- $\alpha(50 \mathrm{ng} / \mathrm{ml})$ for $6 \mathrm{~h}$ (left). Scale bars, $10 \mu \mathrm{m}$. Immunoblot analysis of nuclear and cytoplasm fractions of A549 cells treated with or without TGF- $\alpha$ (50 $\mathrm{ng} / \mathrm{ml})$ for $6 \mathrm{~h}$ (right). (b) Immunoblot analysis of CITED2 expression in A549 cells treated with TGF- $\alpha$ ( $50 \mathrm{ng} / \mathrm{ml}$ ) for the indicated times (top) or with the indicated concentrations of TGF- $\alpha$ for $6 \mathrm{~h}$ (bottom). (c) Immunoblot analysis of total cell lysates from A549 cells infected with scramble control (Sc) or shEGFR lentiviral particles (top). Immunoblot analysis of total cell lysates from A549 cells infected with control or EGFR-L858R-expressing lentiviral particles (bottom). pEGFR, phosphorylated EGFR. (d) Immunoblot analysis of total cell lysates from A549 cells infected with Sc, shCITED2 or shMYC lentiviral particles (left). Immunoblot analysis of total cell lysates from A549 cells infected with control or MYC-expressing lentiviral particles (right). (e) Schematic of the pGL3 vector and the CITED2 promoter reporters CITED2p-pGL3 and CITED2p( $\Delta-717 /-509)$-pGL3, in which MYC-binding site was deleted (top). Luciferase reporter assays with these three vectors were performed in HEK293T cells transfected with pcDNA-MYC or pcDNA control vector (bottom). Results are representative of at least three independent experiments and are expressed as the mean \pm S.D. ${ }^{* *} P<0.001$. (f) Binding of MYC to the CITED2 promoter ( -544 to $-480 \mathrm{bp}$ ) was determined by chromatin immunoprecipitation (ChIP) in A549 cells infected with control or EGFR-L858R-expressing lentiviral particles. ChIP with primers for the site located $5 \mathrm{~kb}$ downstream from CITED2 was performed as a negative control. Results are expressed as the mean \pm S.D. ${ }^{* \star *} P<0.001$. IP, immunoprecipitation 
progression is still elusive. In this study, we show that CITED2 functions as a molecular switch for TGF- $\alpha$ proliferation and TGF- $\beta$ quiescence stimuli by orchestrating MYC-mediated transcription.

\section{Results}

CITED2 expression is induced by TGF- $\alpha$ /EGFR signaling through a MYC-dependent pathway. As TGF- $\alpha / E G F R$
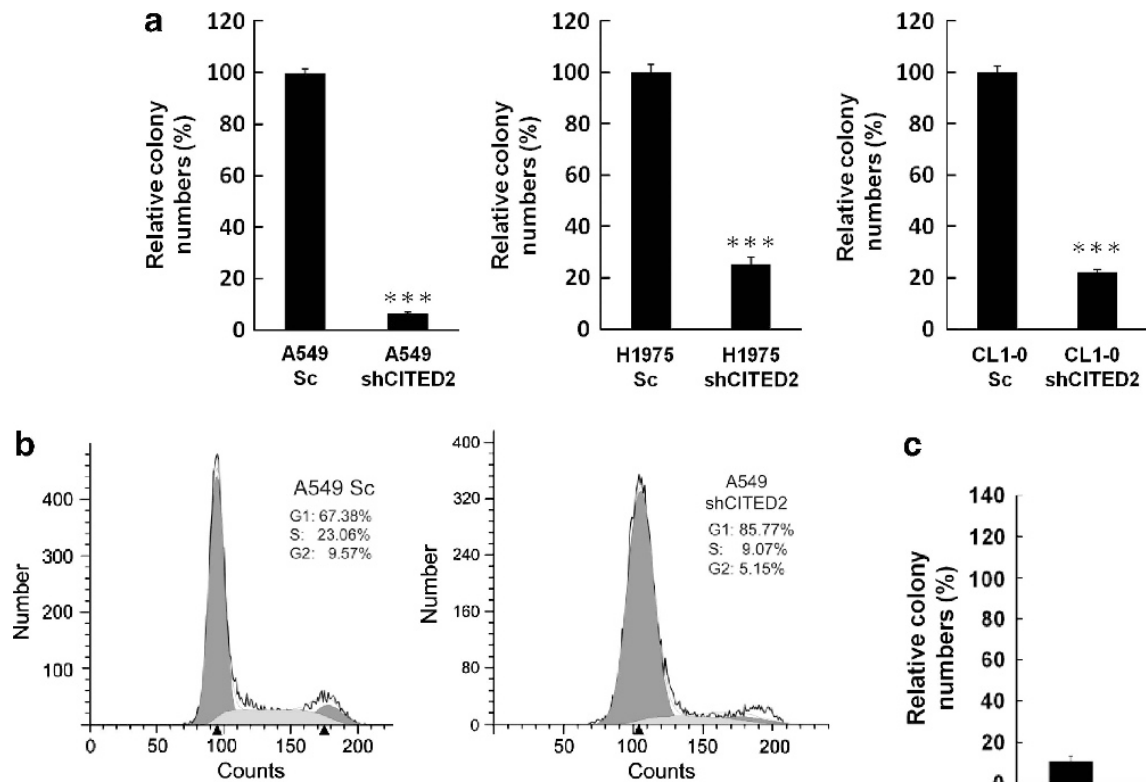

c
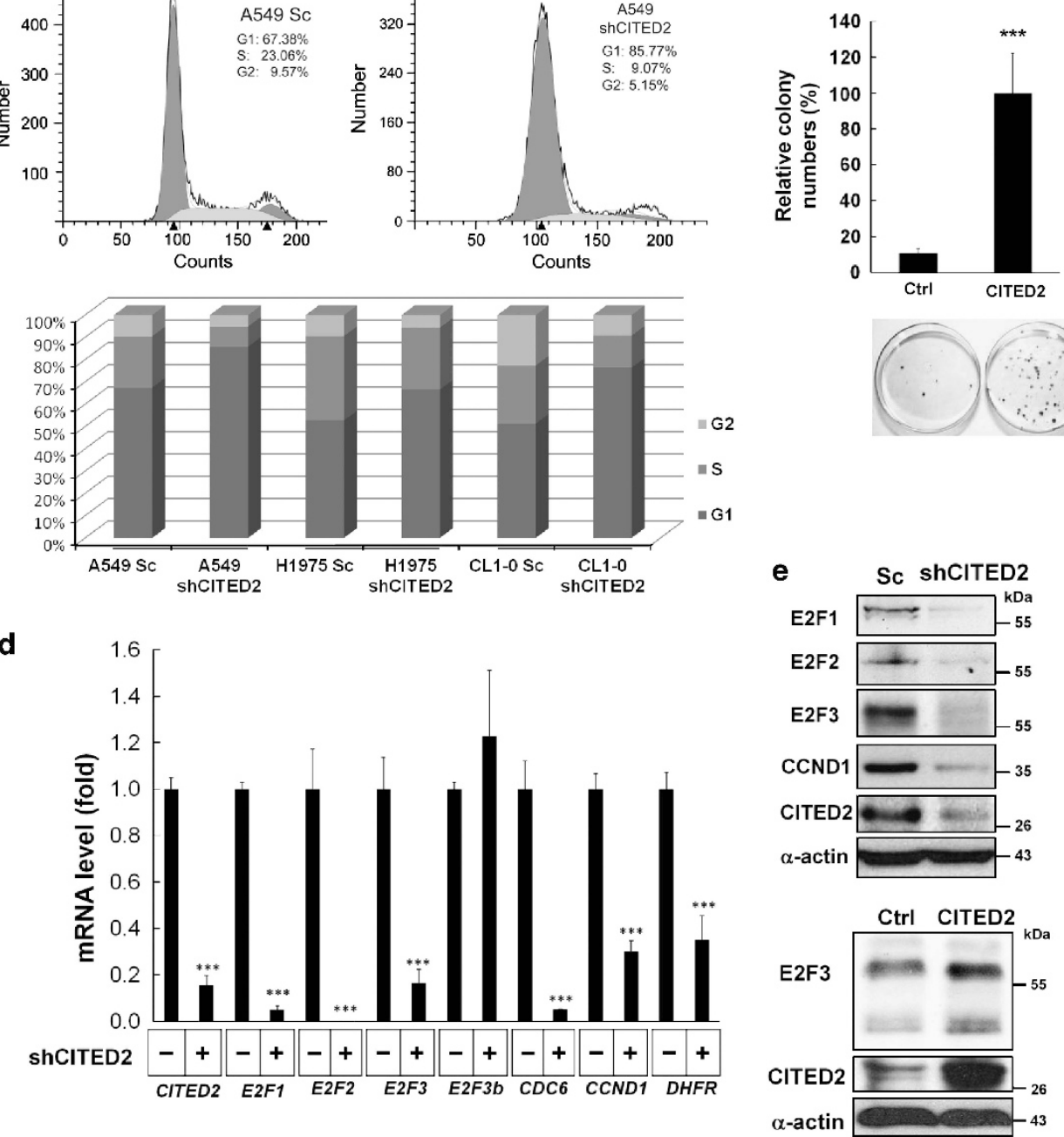

Figure 2 CITED2 regulates E2F3 expression. (a) A549, H1975 and CL1-0 cells were infected with scramble control (Sc) or shCITED2 lentiviral particles for 5 days and subjected to clonogenic assays. Results represent triplicate counts per treatment and are representative of three independent experiments. The relative colony number of Sc cells was set to $100 \%$ and error bars represent mean \pm S.D. ${ }^{* \star} P<0.001$. (b) Flow cytometry cell cycle analysis with propidium iodide staining of A549, H1975 and CL $1-0$ cells infected with Sc or shCITED2 lentiviral particles. A549 data are shown at the top, and the results from all three cell lines are summarized at the bottom. (c) A clonogenic assay of CL1-0 cells infected with control or CITED2-expressing lentiviral particles and maintained under serum-free conditions. The colony number resulting from infection with CITED2 was set to $100 \%$ (top) based on crystal violet staining (bottom). Results are representative of three independent experiments and are expressed as the mean \pm S.D. ${ }^{* * *} P<0.001$. (d) $Q-P C R$ analysis of total RNA from A549 cells infected with Sc $(-)$ or shCITED2 $(+)$ lentiviral particles. Results are representative of three independent experiments and are expressed as the mean \pm S.D. ${ }^{* *} P<0.001$. (e) Immunoblot analysis of total cell lysates from cells as in $\mathrm{d}$ (top). Immunoblot analysis of total cell lysates from CL1-0 cells infected with control or CITED2-expressing lentiviral particles (bottom) 
a

MYC
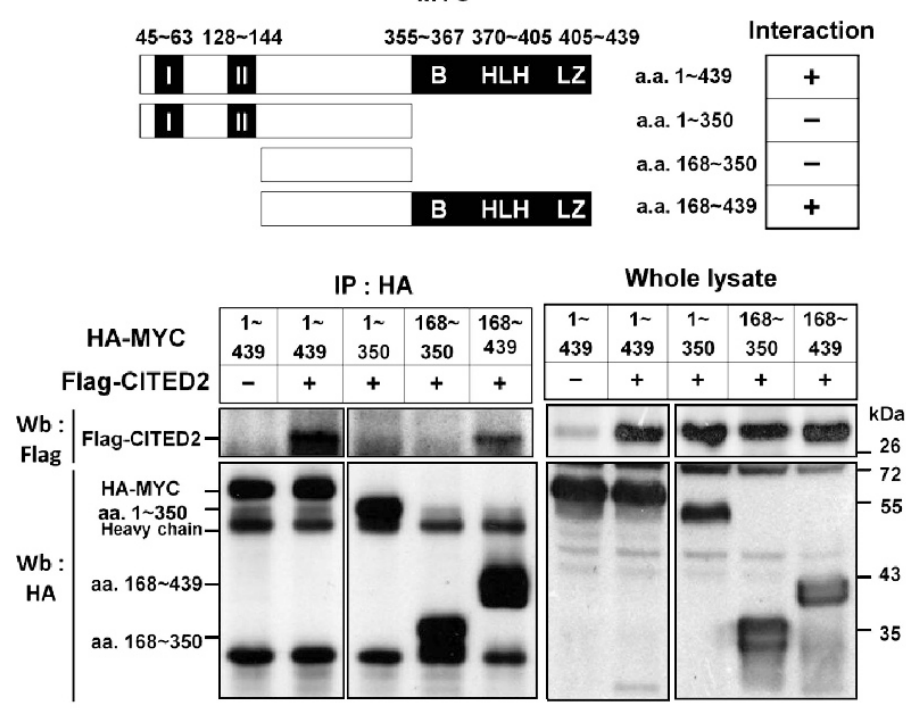

b
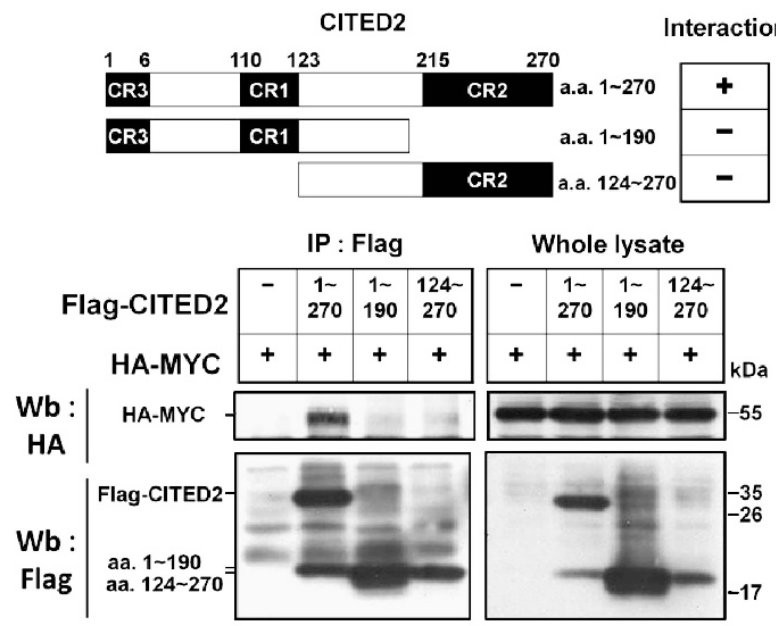

C
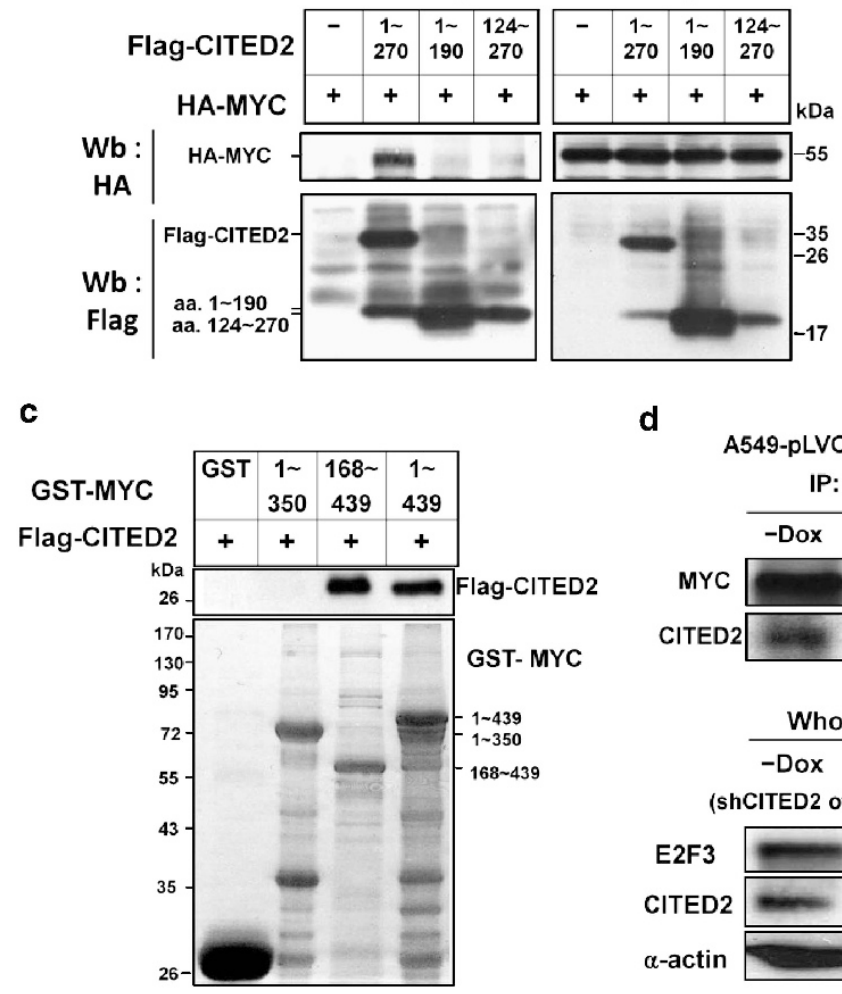

d IP: MYC e
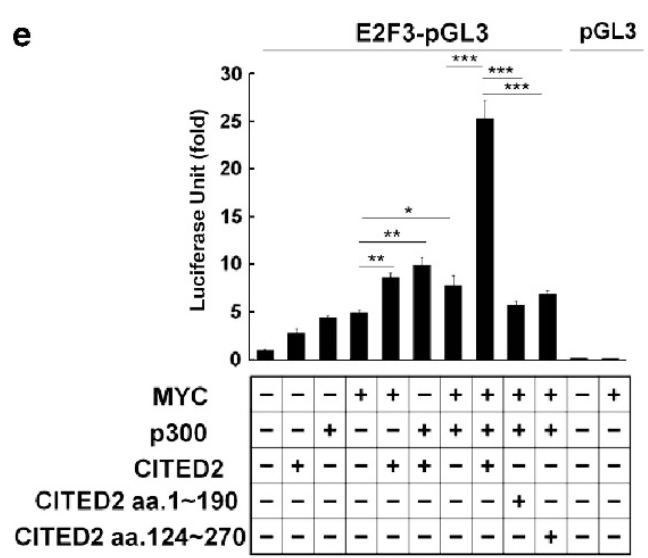

f

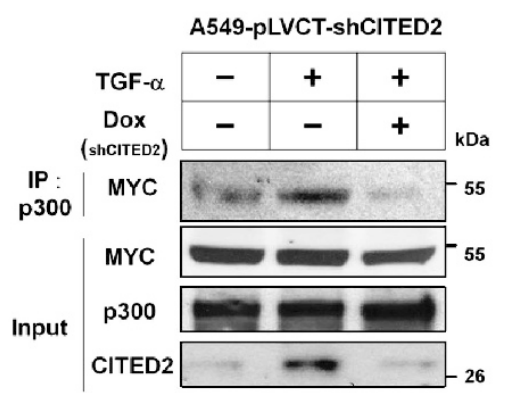

g

A549-pLVCT-shCITED2
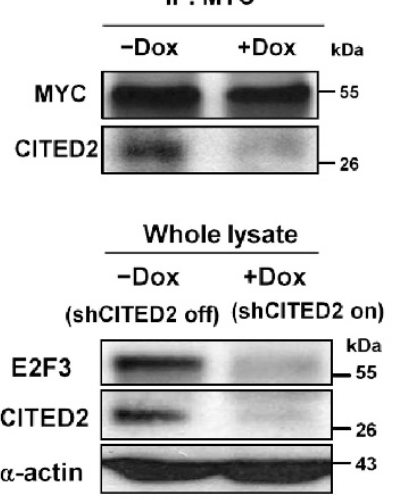
signaling contributes to lung cancer progression ${ }^{3}$ and because CITED2 can be induced by various cytokines, ${ }^{7}$ we examined the effect of TGF- $\alpha / E G F R$ signaling on CITED2 expression in lung cancer cells. Immunofluorescence (IF) staining of A549 lung-cancer cells showed strong nuclear localization of CITED2 after TGF- $\alpha$ induction (Figure 1a). Analysis of CITED2 expression in isolated nuclear and cytoplasmic fractions of A549 cells showed that CITED2 was predominantly localized in the nucleus, and expression of nuclear CITED2 could be further enhanced by TGF- $\alpha$ stimulation (Figure 1a). Immunoblotting demonstrated that TGF- $\alpha$ induced CITED2 expression in A549 cells in a time-dependent and dose-dependent manner (Figure 1b). Furthermore, overexpression of the active L858R mutant EGFR increased the expression of CITED2, as well as MYC (Figure 1c). Knockdown of EGFR by short hairpin RNA (shRNA) against EGFR (shEGFR) attenuated the expression of CITED2 and MYC (Figure 1c). These data demonstrate that TGF- $\alpha / E G F R$ signaling induces CITED2 expression in lung cancer cells.

As MYC is a critical effector of EGFR downstream signaling, ${ }^{24,25}$ we examined the effect of MYC on CITED2 expression. Overexpression of MYC increased CITED2 levels, and knockdown of MYC with MYC-specific shRNA (shMYC) significantly blocked CITED2 expression comparable to that of CITED2-silenced cells, indicating that MYC induces CITED2 expression (Figure 1d). To study the effect of MYC on CITED2 transcription, the 1.3-kb promoter of CITED2 was cloned and used in a promoter reporter assay. The promoter reporter analysis indicated that MYC activated CITED2 transcription (Figure 1e). Analysis of the CITED2 promoter sequence identified a possible MYC-binding site in the CITED2 promoter region (Figure 1e). When the MYC-binding sequence in the CITED2 promoter reporter was deleted, overexpression of MYC did not activate the mutated CITED2 promoter (Figure 1e), suggesting that MYC activates CITED2 transcription through the MYC-binding site. Chromatin immunoprecipitation indicated that the constitutively active EGFR-L858R mutant promoted the binding of MYC to the CITED2 promoter but not to the region $5 \mathrm{~kb}$ downstream from the CITED2 promoter (Figure 1f). Similarly, increased MYC binding to the CITED2 promoter was observed upon TGF- $\alpha$ stimulation (Supplementary Figure S1). Taken together, our data support the notion that TGF- $\alpha$ /EGFR signaling stimulates CITED2 expression via MYC.

CITED2 regulates the E2F-mediated G1/S transition during the cell cycle. To investigate the role of CITED2 in lung cancer cells, CITED2 was knocked down in A549, H1975 and CL1-0 lung cancer cells. CITED2 silencing significantly attenuated the clonogenic activity of all tested lung cancer cells (Figure 2a). We further carried out cell cycle analysis on CITED2-silenced cells and discovered that knockdown of CITED2 caused G1/S cell cycle arrest (Figure 2b and Supplementary Figure S2). As CITED2 can be induced by serum, ${ }^{7}$ to investigate the effect of CITED2 in cellular proliferation, CITED2 was ectopically expressed in CL1-0 cells. Ectopic expression of CITED2 results in sustained growth in serum-free media (Figure 2c). As E2F1, E2F2 and E2F3 are pivotal regulators of G1/S-phase progression, we performed quantitative RT-PCR (Q-PCR) analysis of E2F1, E2F2 and E2F3 expression in CITED2 knockdown cells. We found that E2F1, E2F2 and E2F3 and its downstream targets such as CDC6, CCND1 and DHFR were downregulated in CITED2-silenced A549 cells; in contrast, the short isoform of E2F3 (referred to as E2F3b) was not affected by CITED2 silencing (Figure 2d). Western blotting further demonstrated that CITED2 silencing inhibited E2F1, E2F2 and E2F3 expression in A549 cells (Figure 2e). Overexpression of CITED2 not only promoted clonogenic growth but also enhanced the amount of E2F3 in CL1-0 cells (Figure $2 \mathrm{c}$ and $\mathrm{e}$ ). These results indicate that CITED2 controls cell cycle progression.

CITED2 interacts with MYC and potentiates MYC transactivation activity by recruiting p300. MYC directly regulates the expression of E2F1-3. ${ }^{16,26}$ As CITED2 regulates E2F1-3 levels, it is possible that CITED2 regulates E2F1-3 by modulating MYC-mediated transcription. To test this hypothesis, we first investigated whether CITED2 interacts with MYC using co-immunoprecipitation. We constructed MYC truncation mutants to map the CITED2interacting region in MYC. CITED2 interacted with full-length MYC (residues 1-439) and residues 168-439 of MYC, but not with residues $1-350$ or $168-350$, indicating that CITED2 interacts with the $\mathrm{C}$ terminus but not the $\mathrm{N}$ terminus of MYC (Figure 3a). Conversely, CITED2 truncation mutants were

Figure 3 CITED2 promotes MYC-mediated transactivation of E2F3. (a) Schematic illustration of the functional domains in wild-type MYC and MYC deletion mutants (top). B, HLH and LZ represent basic, helix-loop-helix and leucine zipper domains, respectively. Western blotting analysis (Wb) of hemagglutinin (HA)-immunoprecipitated products or whole-cell lysates from HEK293T cells transfected with pFlag-CMV2-CITED2 (Flag-CITED2) and pcDNA-HA-MYC (HA-MYC) or HA-tagged MYC deletion mutants (bottom). (b) Schematic illustration of the conserved regions (CR1, CR2 and CR3) in wild-type CITED2 and CITED2 deletion mutants (top). Western blotting analysis of Flag-immunoprecipitated products or whole-cell lysates from HEK293T cells transfected with HA-MYC and Flag-CITED2 or Flag-tagged CITED2 deletion mutants (bottom). (c) In vitro-transcribed and -translated Flag-CITED2 was mixed with GST-tagged MYC deletion mutants and subjected to a GST pull-down assay. Immunoblotting and Coomassie blue staining show the resulting expression. (d) A549-pLVCT-shCITED2 cells were treated with or without Dox ( $\mu \mathrm{g} / \mathrm{ml})$ for $24 \mathrm{~h}$ and subjected to western blotting to monitor CITED2 and E2F3 expression (bottom). Whole-cell lysates were immunoprecipitated with antibodies against endogenous MYC followed by immunoblotting for MYC and CITED2 (top). (e) A luciferase reporter assay for E2F3 promoter activity was carried out in HEK293T cells transfected with pcDNA-MYC, pcDNA-p300 and/or Flag-CITED2 deletion mutants together with the E2F3 promoter reporter E2F3-pGL3 or pGL3. Results are representative of at least three independent experiments and are expressed as the mean \pm S.D. ${ }^{*} P<0.05 ;{ }^{* *} P<0.01 ;{ }^{* *} P<0.001$. (f) A549-pLVCT-shCITED2 cells were treated with or without TGF- $\alpha$ ( $\left.50 \mathrm{ng} / \mathrm{ml}\right)$ for $8 \mathrm{~h}$ in the presence or absence of Dox $(1 \mu \mathrm{g} / \mathrm{ml})$ and monitored for expression of p300, MYC and CITED2 in cells (input). Binding of endogenous p300 to MYC was determined by co-immunoprecipitation with antip300, followed by immunoblotting for MYC. (g) E2F3 promoter sequences from mouse and human showing conserved MYC-binding sequences (top). A549-pLVCTshCITED2 cells were treated with or without TGF- $\alpha(50 \mathrm{ng} / \mathrm{ml})$ for $8 \mathrm{~h}$ in the presence or absence of Dox $(1 \mu \mathrm{g} / \mathrm{ml})$ and were subjected to ChIP analysis with the E2F3 promoter (middle) or with $5 \mathrm{~kb}$ downstream from the promoter (bottom) with antibodies against MYC, CITED2 and p300. Results are expressed as the mean \pm S.D. ${ }^{* \star *} P<0.001$ 
also constructed to map the MYC-interacting region of CITED2. MYC interacted with full-length CITED2 but not with the $\mathrm{N}$ - or $\mathrm{C}$-terminal deletion mutants (Figure $3 \mathrm{~b}$ ). These results indicate a specific interaction between $M Y C$ and CITED2. To further confirm the physical interaction between CITED2 and MYC, a glutathione S-transferase (GST) pulldown assay was performed. GST-tagged MYC deletion constructs were expressed in vitro, and full-length CITED2 was transcribed and translated in vitro. CITED2 interacted with GST-MYC residues 168-439 but not with GST-MYC residues 1-350 (Figure $3 c$ ). These results indicate that the C-terminal region of MYC is necessary for the interaction with CITED2.

To obtain cells in which endogenous CITED2 can be manipulated, we generated A549 cells that stably express doxycycline (Dox)-inducible shRNA against CITED2 (A549-pLVCT-shCITED2). Among E2Fs, E2F3 is highly expressed in lung cancer; ${ }^{18}$ therefore, we focused on E2F3 expression. After endogenous CITED2 was knocked down, the expression of E2F3 decreased (Figure 3d). Moreover, endogenous CITED2 was co-immunoprecipitated by antiMYC, supporting a specific interaction between MYC and CITED2 (Figure 3d).

As CITED2 interacts with MYC (which regulates E2F3 transcription), we performed a promoter reporter assay to investigate the role of CITED2 in MYC-mediated E2F3 expression. We found that MYC stimulated E2F3 promoter reporter activity, and CITED2 promoted MYC-mediated transactivation of the E2F3 promoter (Figure $3 e$ ). As CITED2 interacts with $\mathrm{p} 300,{ }^{14}$ we further investigated whether CITED2 promoted MYC-mediated transactivation by recruiting p300. Through co-immunoprecipitation analysis with antibodies against p300, we found that treating A549pLVCT-shCITED2 cells with TGF- $\alpha$ induced CITED2 expression and enhanced the interaction between p300 and MYC; this interaction was attenuated by knockdown of CITED2 with Dox treatment (Figure 3f). Moreover, a reporter assay indicated that the expression of CITED2, but not of $\mathrm{N}$ - or C-terminal deletion mutants of CITED2, promoted MYCmediated E2F3 promoter activity in the presence of ectopic p300 (Figure 3e). These data suggest that CITED2 interacts with MYC and recruits p300 to activate E2F3 expression. Myc binding sequences have been identified in the mouse E2f3 promoter. ${ }^{16}$ By comparing the sequences between the mouse and human E2F3 promoters, we identified putative MYCbinding sequences in the human E2F3 promoter (Figure $3 \mathrm{~g}$ ). Following chromatin immunoprecipitation analysis with antibodies against MYC, CITED2 and p300, we discovered that increased CITED2, MYC and p300 bound to the E2F3 promoter upon TGF- $\alpha$ stimulation, and knockdown of CITED2 not only inhibited CITED2 but also attenuated p300 binding to the E2F3 promoter. (Figure $3 \mathrm{~g}$ ). These data support that CITED2 binds to MYC and recruits p300 to the E2F3 promoter to enhance E2F3 expression.

TGF- $\beta$ downregulates CITED2, leading to cellular quiescence. In contrast to TGF- $\alpha$, TGF- $\beta$ promotes quiescence in epithelial cells, and TGF- $\beta$-mediated downregulation of MYC is thought to be responsible for TGF- $\beta$-induced cellular quiescence. $^{23}$ We examined the effects of TGF- $\beta$ on the clonogenic growth of lung adenocarcinoma cells. TGF- $\beta$ mediated cellular quiescence was observed in A549 and H1975 cells but not in CL1-0 cells (Figure 4a). As CITED2silencing induced cellular quiescence of these cells (Figures $2 a$ and $b$ ), we also examined whether CITED2 expression is affected by TGF- $\beta$ stimulation. Upon TGF- $\beta$ treatment, nuclear CITED2 was decreased in A549 cells (Figure 4b). Furthermore, TGF- $\beta$ downregulated both MYC and CITED2 but induced $\mathrm{p} 21^{\mathrm{CIP} 1}$ in A549 cells in a time-dependent manner (Figure 4b). The Q-PCR data supported that TGF- $\beta$ induced MYC downregulation in A549 cells; however, TGF$\beta$-mediated downregulation of $M Y C$ was not observed in H1975 cells (Figure 4c). Interestingly, CITED2 expression was inhibited by TGF- $\beta$ in both A549 and H1975 cell lines, which responded to TGF- $\beta$-induced cellular quiescence (Figures $4 \mathrm{a}$ and $\mathrm{c}$ ). As defective expression of TGF- $\beta$ receptors (TGFBRI and TGFBRII) is found in cells from patients with lung cancer, ${ }^{5,6}$ expression of TGFBRI and TGFBRII messenger RNA (mRNA) was investigated in these cells. Absolute Q-PCR analysis and western blot indicated loss of TGFBRII in CL1-0 cells (Figure 4d). Furthermore, methylation status of the TGFBRII promoter in these lung cancer cells was analyzed by bisulfite sequencing. The TGFBRII promoter in CL1-0 cells, but not in A549 and H1975 cells, was highly methylated (Supplementary Figure S3). To investigate whether downregulation of CITED2 is responsible for TGF- $\beta$-mediated cellular quiescence, CITED2 was ectopically expressed in $\mathrm{H} 1975$ cells, followed by TGF- $\beta$ stimulation. We observed that CITED2-overexpressing $\mathrm{H} 1975$ cells were resistant to TGF- $\beta$-mediated cell growth arrest (Figure $4 \mathrm{e}$ ). These data suggest that downregulation of CITED2 contributes to TGF- $\beta$-mediated cellular quiescence.

CITED2 regulates the $\mathrm{p} 21^{\mathrm{CIP} 1}$ senescence pathway by enhancing the MYC-histone deacetylase 1 (HDAC1) interaction. As $p 21^{C I P 1}$ is induced by TGF- $\beta$ to drive cellular quiescence, ${ }^{27}$ which is mediated by downregulation of CITED2, we examined the expression of $p 21^{C I P 1}$ and its correlation with CITED2 levels in TGF- $\beta$-treated cells. We found that both protein and mRNA levels of $p_{21}{ }^{C I P 1}$ were negatively correlated with CITED2 expression after TGF- $\beta$ stimulation in A549 cells (Figure $4 b$ and Figure 5a). In addition, $p 21^{C I P 1}$ mRNA was induced by TGF- $\beta$ in H1975 cells; moreover, overexpression of CITED2 in H1975 cells not only rendered cells resistant to TGF- $\beta$-mediated growth arrest but also attenuated $p 21^{C I P 1}$ expression (Figure $4 \mathrm{~d}$ and Figure $5 b$ ). These results suggest a possible role for CITED2 in the regulation of the $\mathrm{p} 21^{\mathrm{ClP} 1}$ senescence pathway.

As TGF- $\beta$ induces $\mathrm{p} 21^{\mathrm{CIP} 1}$ to establish cellular senescence, ${ }^{28}$ we investigated the role of CITED2 in the senescence of lung cancer cells. Whereas TGF- $\beta$ downregulated CITED2 and induced senescence-associated $\beta$-galactosidase (SA- $\beta$-gal) in A549 cells (Figures 5 a and c), we found that knockdown of CITED2 increased the activity of SA- $\beta$-gal in A549 with a senescence phenotype, including a flattened and enlarged morphology (Figures $5 d$ and $e$ and Supplementary Figure S4). Additionally, CITED2 silencing in A549 cells induced expression of $p 21^{\mathrm{CIP} 1}$ mRNA (Figure $5 \mathrm{e}$ and Supplementary Figure S4). These data support that 
CITED2 regulates $p 21^{C I P 1}$ expression. As $p 21^{C I P 1}$ can be induced by a p53-dependent or p53-independent pathway, ${ }^{29}$ CITED2 was knocked down in both A549 (wild-type p53) and H1299 (p53 null) cells, followed by immunoblotting to detect p21 ${ }^{\mathrm{CIP} 1}$ expression. We observed that p21 ${ }^{\mathrm{CIP} 1}$ was induced in both cell types regardless of p53 expression (Figure 5f), suggesting that $\mathrm{p} 21^{\mathrm{CIP} 1}$ induction in CITED2-silenced cells is through a p53-independent pathway. To investigate whether increased $\mathrm{p}_{21} \mathrm{CIP}^{\mathrm{C} 1}$ expression is responsible for senescence in CITED2-silenced cells, both $\mathrm{p} 21^{\mathrm{CIP} 1}$ and CITED2 were knocked down in A549 cells and the cells were then subjected to the SA- $\beta$-gal assay (Figure $5 \mathrm{~g}$ ). Although CITED2-silencing induced cellular senescence, knockdown of $\mathrm{p} 21^{\mathrm{CIP} 1}$ rescued the CITED2-silenced cells from senescence, indicating that

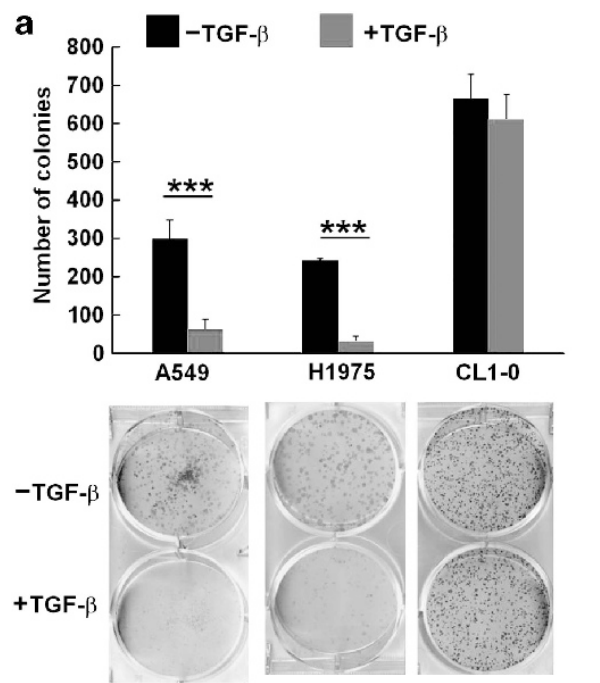

b
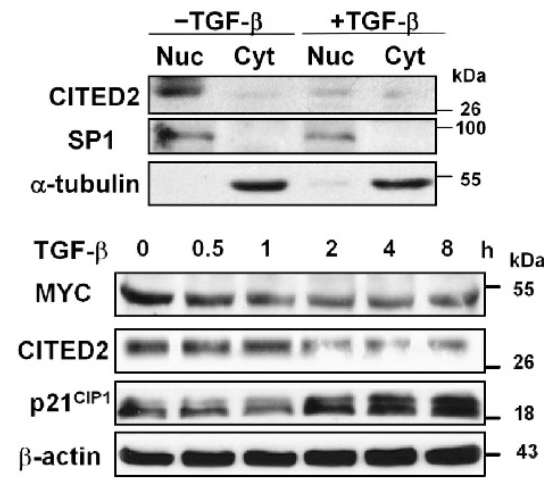

c

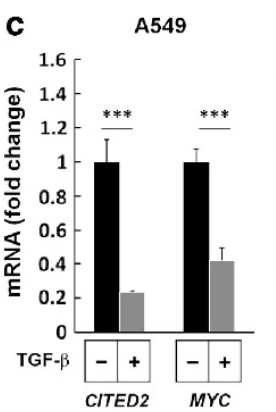

d

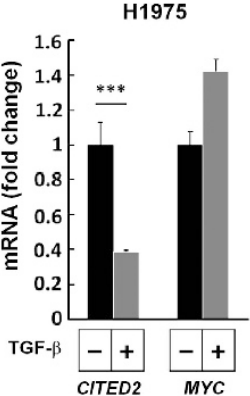

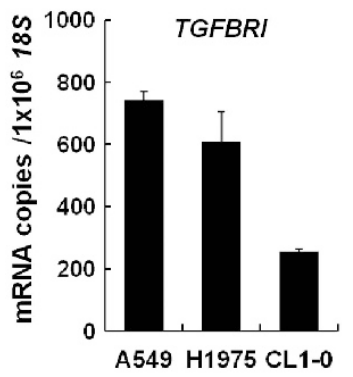
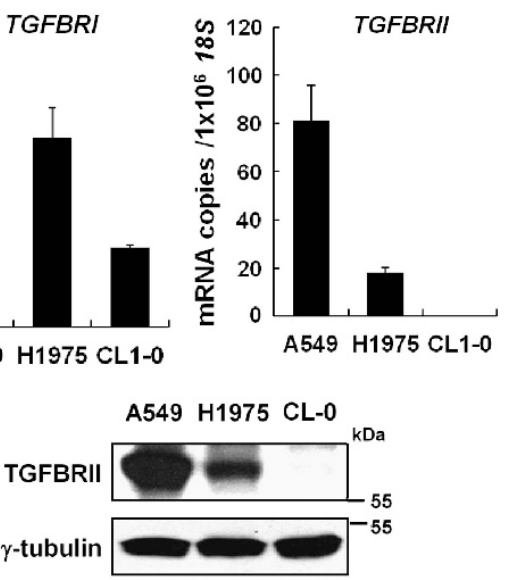

e

-TGF- $\beta \quad+$ TGF- $\beta$
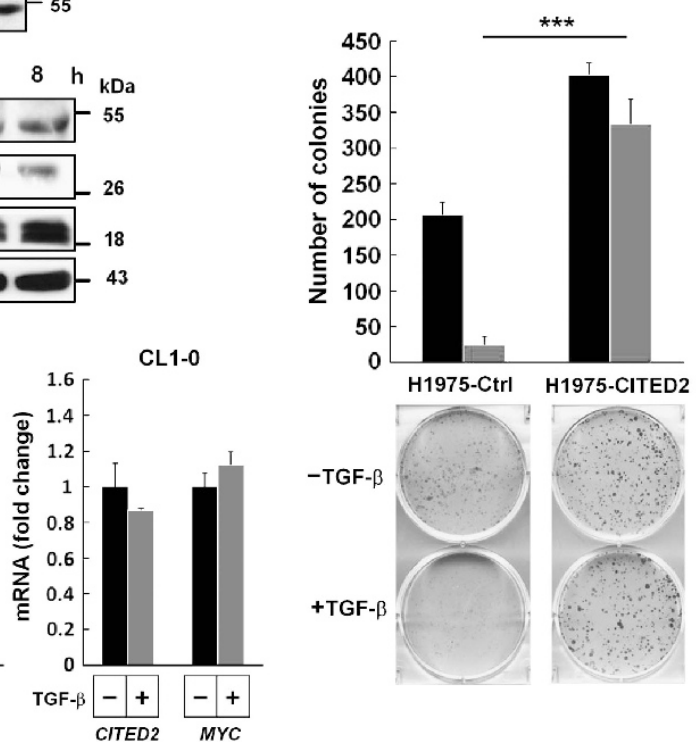

Figure 4 CITED2 downregulation contributes to TGF- $\beta$-induced quiescence. (a) Clonogenic assays of A549, H1975 and CL1- 0 cells treated with or without TGF- $\beta$ (2 ng/ml) for 14 days (top) and stained with crystal violet (bottom). Results are representative of three independent experiments. Error bars represent mean \pm S.D. ${ }^{* * *} P<0.001$. (b) Immunoblot analysis of nuclear and cytoplasm fractions of A549 cells treated with or without TGF- $\beta(2 \mathrm{ng} / \mathrm{ml})$ for $6 \mathrm{~h}$ (top). Immunoblot analysis of total cell lysates from A549 cells treated with TGF- $\beta(2 \mathrm{ng} / \mathrm{ml})$ for the indicated times (bottom). (c) Q-PCR analysis for the expression of MYC and CITED2 mRNA in A549, H1975 and CL1-0 cells treated with $(+)$ or without $(-)$ TGF- $\beta(2 \mathrm{ng} / \mathrm{ml})$ for $6 \mathrm{~h}$ (bottom). Results are representative of at least three independent experiments and are expressed as the mean \pm S.D. ${ }^{* * \star} P<0.001$. (d) Absolute Q-PCR analysis of TGFBRI (upper left) and TGFBRII (upper right) mRNA in A549, H1975 and CL1-0 cells. Immunoblot analysis of total cell lysates from A549, H1975 and CL1-O cells (lower). (e) H1975 cells were infected with control or CITED2-expressing lentiviral particles for 5 days followed by treatment with or without TGF- $\beta$ ( $2 \mathrm{ng} / \mathrm{ml}$ ) for 14 days. Clonogenic assays were carried out (top) by staining with crystal violet (bottom). Results are representative of three independent experiments and are expressed as the mean \pm S.D. ${ }^{* *} P<0.001$ 


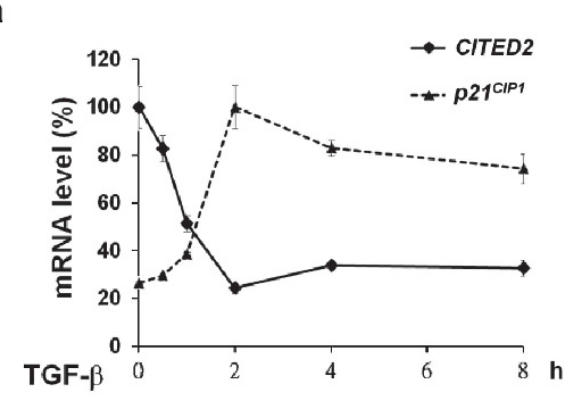

d
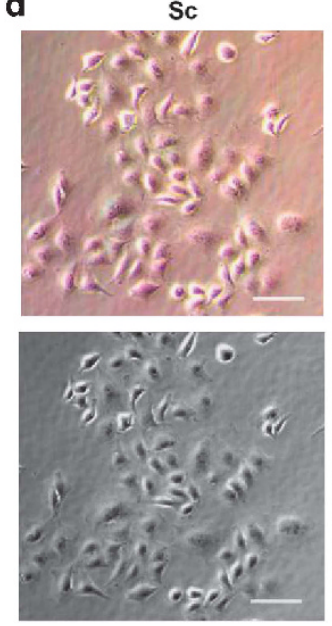

g

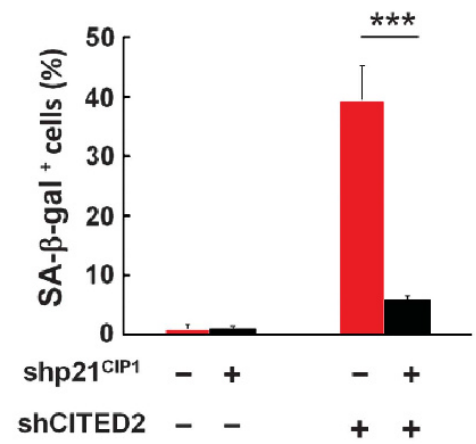

ShCITED2
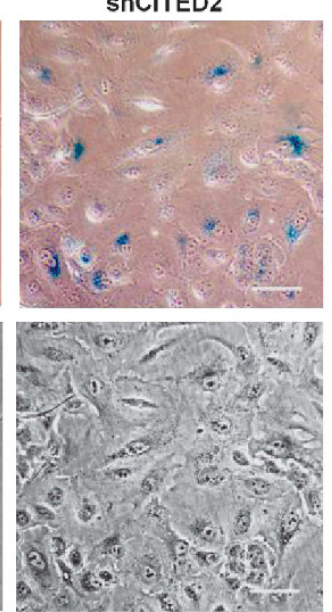

**
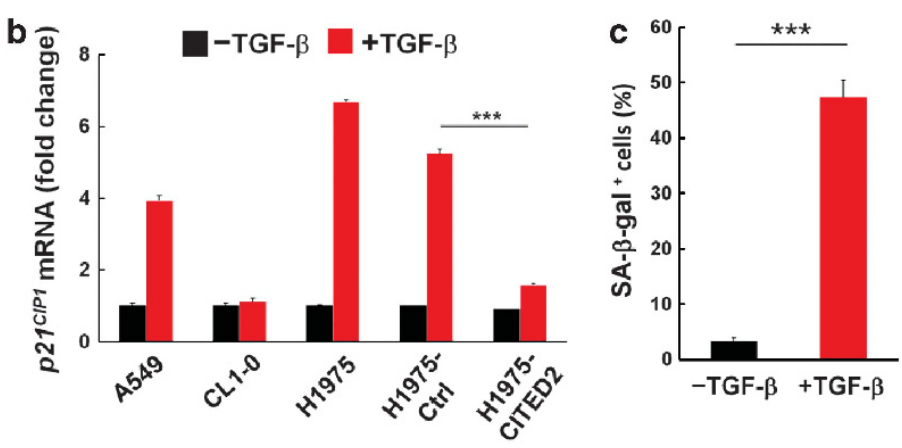

e
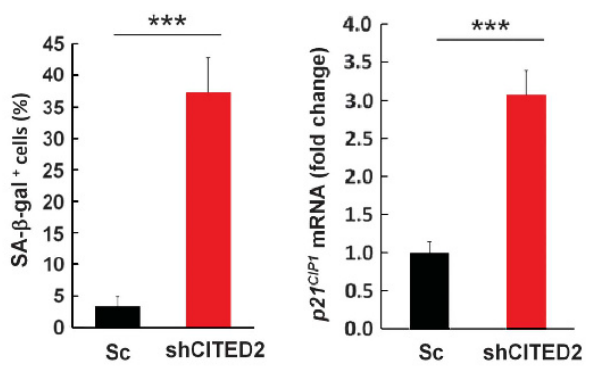

f

$\frac{A 549}{\text { Sc } \text { ShCITED2 }}$

CITED2

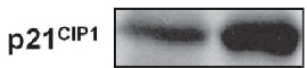

$\beta$-actin

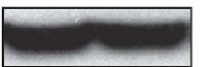

i

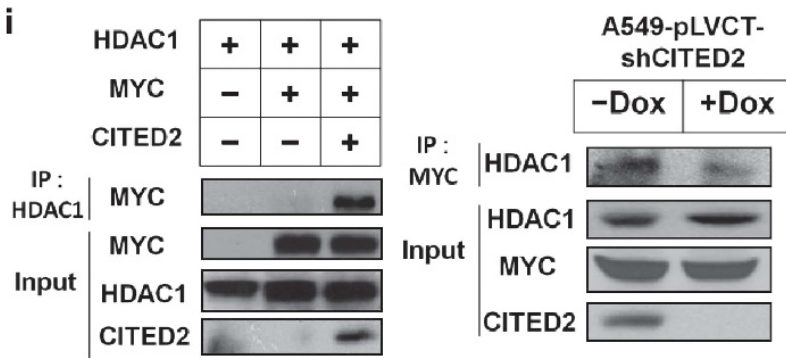

h

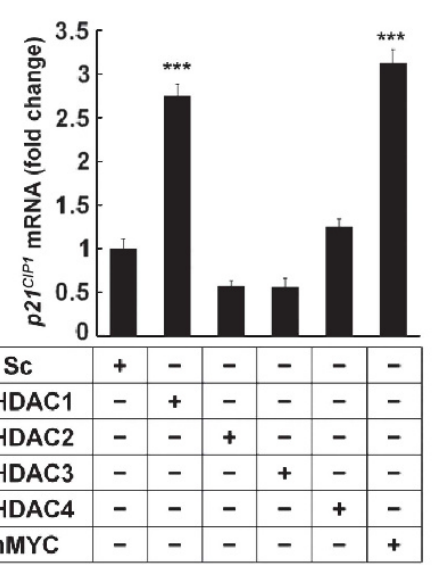

j

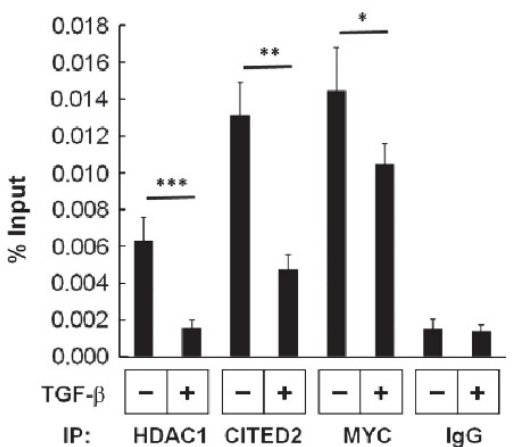

p21 ${ }^{\text {CIP1 }} 5 \mathrm{~kb}$ down

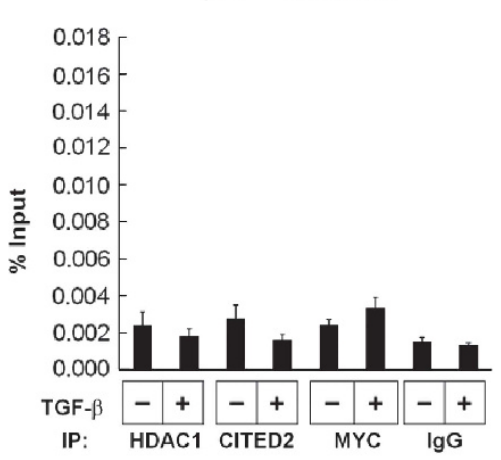


p21 ${ }^{\mathrm{CIP} 1}$ mediates the senescence (Figure $5 \mathrm{~g}$ ). MYC and HDACs inhibit p21 ${ }^{\mathrm{CIP} 1}$ expression; ${ }^{23,30}$ consistent with other reports, we found that knockdown of MYC or HDAC1 induced p2 ${ }^{\mathrm{CIP} 1}$ expression (Figure $5 \mathrm{~h}$ ). Furthermore, we observed that CITED2 interacted with HDAC1 (Supplementary Figure S5). As CITED2 interacts with MYC and HDAC1, it is possible that CITED2 mediates $\mathrm{p} 21^{\mathrm{CIP} 1}$ suppression by recruiting HDAC1 to the MYC-interacting complex. To test this hypothesis, we first investigated whether CITED2 promotes MYC interaction with HDAC1. Through co-immunoprecipitation, we found that ectopically expressed MYC in HEK293T cells did not interact with HDAC1, but co-expression of CITED2 enriched the MYC-HDAC1-interacting complex (Figure 5i). Similarly, the endogenous MYC-HDAC1-interacting complex was observed through a co-immunoprecipitation assay; knockdown of CITED2 decreased the formation of the MYC-HDAC1-interacting complex (Figure 5i). These data support that CITED2 promotes MYC interactions with HDAC1. Moreover, chromatin immunoprecipitation showed that although both CITED2 and HDAC1 were recruited to the MYC-binding region of the $p 21^{C I P 1}$ promoter, TGF- $\beta$ stimulation attenuated the binding of CITED2 and HDAC1 to the $p 21^{C I P 1}$ promoter (Figure 5j). These data demonstrate that CITED2 modulates MYC-mediated $p 21^{C I P 1}$ suppression by recruiting HDAC1.

CITED2 promotes the growth of lung tumor xenografts. To investigate the role of CITED2 in vivo, we injected CITED2-overexpressing or control A549 cells subcutaneously into athymic nude mice. We examined tumor volume over time and observed that CITED2 overexpression significantly enhanced tumor growth rates and increased tumor weight (Figure 6a). To evaluate the significance of endogenous CITED2 in tumor formation, we used A549-pLVCT-shCITED2 cells in a xenograft animal model. When palpable tumor bulges were observed in the host mice, shCITED2 was induced in the xenograft tumors through Dox treatment (Figure 6b). We found that knockdown of endogenous CITED2 with Dox treatment caused significant tumor shrinkage (Figure 6b). To examine whether CITED2 could function as a therapeutic target for lung cancer treatment, luciferase/enhanced green fluorescent protein
(eGFP)-labeled A549 cells with Dox-inducible shCITED2 were orthotopically implanted into the lungs of nude mice and monitored by live imaging. We observed that knockdown of CITED2 significantly decreased the growth of orthotopically implanted lung tumors and increased the overall survival rate of recipient mice ( $P=0.0004$; Figure $6 \mathrm{c})$. To examine the expression of CITED2, MYC, E2F3 and p2 $1^{\mathrm{CIP} 1}$ during tumorigenesis, orthotopically implanted lung tumors were excised and examined for tumor growth using fluorescence microscopy (upper images) and analyzed by immunohistochemistry (IHC) (lower images) for CITED2, MYC, E2F3 and p21 ${ }^{\mathrm{CIP} 1}$ expression (Figure $6 \mathrm{~d}$ ). We found that CITED2, MYC and E2F3 were highly expressed in neoplastic cells of lung xenografts (Figure 6d). In contrast, normal alveolar ducts and alveoli were observed in the CITED2 knockdown xenografts, and high $\mathrm{p} 21^{\mathrm{CIP} 1}$ expression was detected in normal alveolar cells (Figure 6d). These data support that CITED2 regulates lung tumor development.

Poor overall survival rate of patients with NSCLC was associated with expression of the CITED2/MYC/E2F3/ p21 ${ }^{\mathrm{CIP} 1}$ pathway. The above results suggest that the CITED2/MYC/E2F3/p21 ${ }^{\mathrm{CIP} 1}$ pathway is activated by TGF- $\alpha$ but inhibited by TGF- $\beta$ cytokine signaling. As abnormal cytokine signaling such as EGFR activation and TGFBRII silencing occurs in most patients with NSCLC, ${ }^{2,5}$ we studied the levels of MYC, CITED2, E2F3 and p21 CiP1 proteins by IHC of a panel of specimens from 72 individuals with NSCLC (Figure 7a). Patient characteristics are summarized in Supplementary Table S1. Kaplan-Meier survival analysis was then conducted to determine the prognostic significance of CITED2, MYC, E2F3 and p21 ${ }^{\mathrm{CIP} 1}$ expression in patients with NSCLC. First, the results showed that the CITED2positive cases were associated with a considerably worse overall survival rate as compared with CITED2-negative ones (Figure 7b). Second, patients with lower MYC expression had a better survival prognosis as compared with patients who expressed high levels of MYC (Figure 7c). Third, patients with high E2F3 expression had a poor survival prognosis (Figure $7 d$ ). Fourth, the overall survival rate of p21-positive patients was higher than that of p21-negative patients (Figure 7e). Finally, CITED2 +/MYC +/E2F3 +

\footnotetext{
Figure 5 CITED2 modulates MYC-mediated suppression of the p21 ${ }^{\mathrm{CIP} 1}$ senescence pathway. (a) Q-PCR analysis of CITED2 and p21 ${ }^{\mathrm{CIP1}}$ mRNA in A549 cells treated with TGF- $\beta$ ( $2 \mathrm{ng} / \mathrm{ml}$ ) for the indicated times. (b) Q-PCR analysis of $p 21^{\mathrm{CIP} 1}$ mRNA in A549, CL1-0, H1975 and H1975 with empty vector (Ctrl) and H1975-CITED2 cells treated with or without TGF- $\beta(2 \mathrm{ng} / \mathrm{ml})$ for $6 \mathrm{~h}$. Results are representative of at least three independent experiments and are expressed as the mean \pm S.D. ${ }^{* * \star} P<0.001$. (c) A549 cells treated with or without TGF- $\beta(2 \mathrm{ng} / \mathrm{ml})$ for 5 days were subjected to a senescence assay by measuring SA- $\beta$-gal positive cells. Results are representative of three independent experiments and are expressed as the mean \pm S.D. ${ }^{* \star *} P<0.001$. (d) The photographs show SA- $\beta$-gal activity at pH 6.0, stained in blue color (upper images), and changes in morphology (lower images) of A549 cells 5 days after infection with pLKO.1-Scramble (Sc) or pLKO.1-shCITED2. Scale bars, $20 \mu$ m. (e) SA- $\beta$-gal positive cells in $\mathrm{d}$ were quantified (left). p21 $1^{\mathrm{ClP1}}$ mRNA in A549 cells infected with pLKO.1-Sc or pLKO.1-shCITED2 was determined by Q-PCR (right). Results are representative of three independent experiments and are expressed as the mean \pm S.D. ${ }^{* *} P<0.001$. (f) CITED2 and p21 ${ }^{\mathrm{CIP} 1}$ were analyzed by immunoblotting in A549 (left)

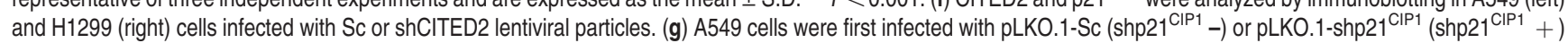
and were subsequently infected with (shCITED2 + ) or without (shCITED2 -) pLKO.1-shCITED2 and were assayed as in c. Results are representative of three independent experiments and are expressed as the mean \pm S.D. ${ }^{* * *} P<0.001$. (h) Q-PCR analysis of total RNA derived from A549 cells infected with Sc, shHDAC1, shHDAC2, shHDAC3, shHDAC4 or shMYC lentiviral particles. Results are representative of at least three independent experiments and are expressed as the mean \pm S.D. ${ }^{* \star *} P<0.001$. (i) Immunoblotting of HDAC1-immunoprecipitated products or whole-cell lysates from HEK293T cells transfected with pcDNA-HDAC1, pcDNA-HA-MYC and/or Flag-CITED2 (left). A549-pLVCT-shCITED2 cells were treated with or without Dox $(1 \mu \mathrm{g} / \mathrm{ml})$ for $24 \mathrm{~h}$, and the expression of HDAC1, MYC and CITED2 in cells was monitored. Binding of endogenous HDAC1 to MYC was determined by co-immunoprecipitation with anti-HDAC1, followed by immunoblotting for MYC (right). (j) Binding of MYC, CITED2 and HDAC1 to the $p 21^{C I P 1}$ promoter region ( -301 to $-242 \mathrm{bp}$ ) and the $5 \mathrm{~kb}$ downstream region was analyzed by chromatin immunoprecipitation with antibodies against MYC, CITED2 and HDAC1 in A549 cells treated with or without TGF- $\beta$ ( $2 \mathrm{ng} / \mathrm{ml})$ for $6 \mathrm{~h}$. Results are expressed as the mean \pm S.D. ${ }^{*} P<0.05,{ }^{* \star} P<0.01,{ }^{* \star *} P<0.001$
} 


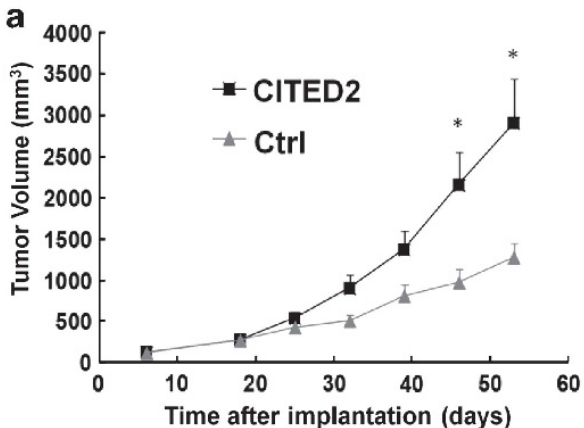

b
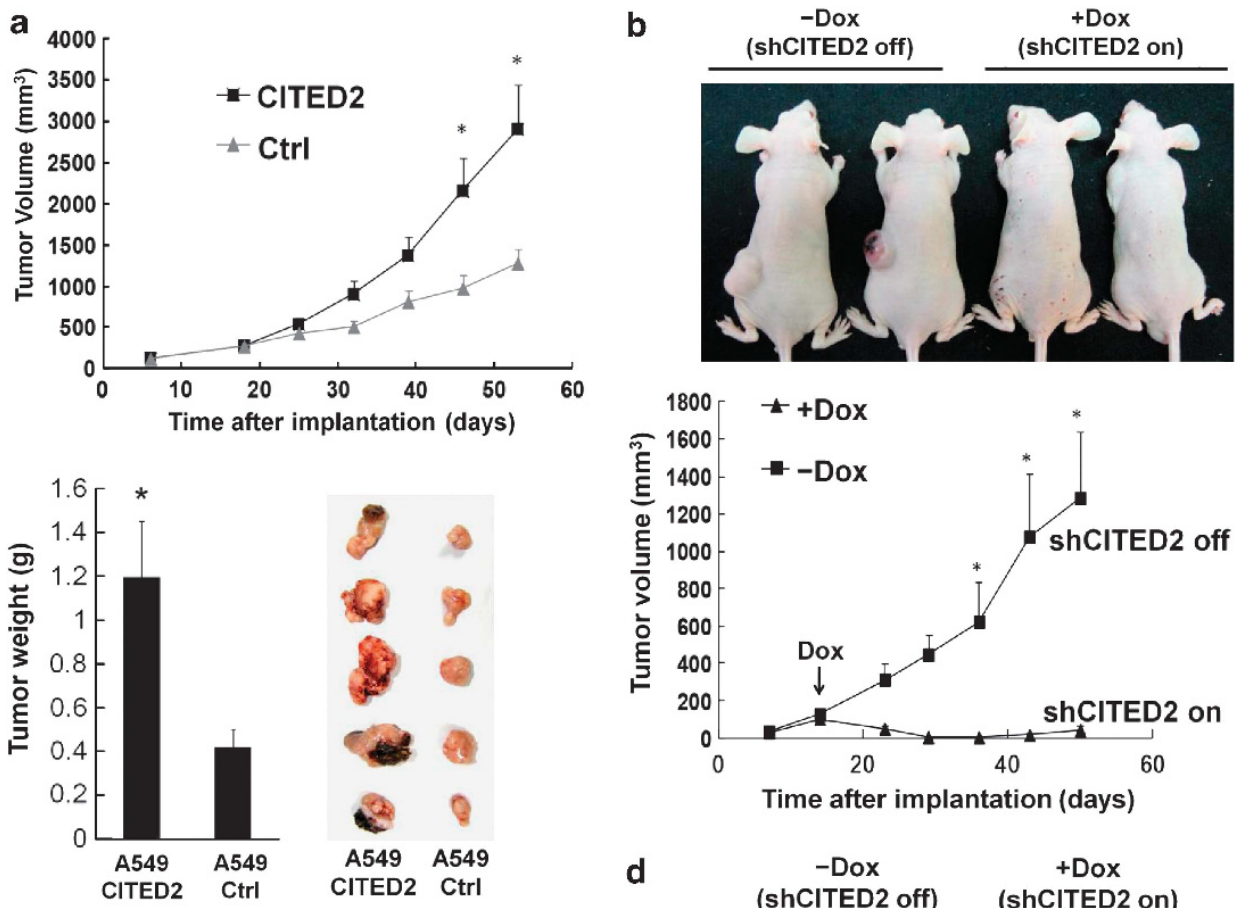

C

A549-pLVCT-shCITED2

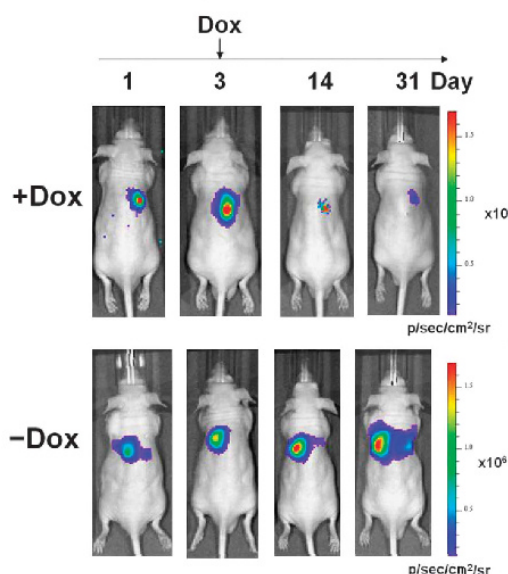

d

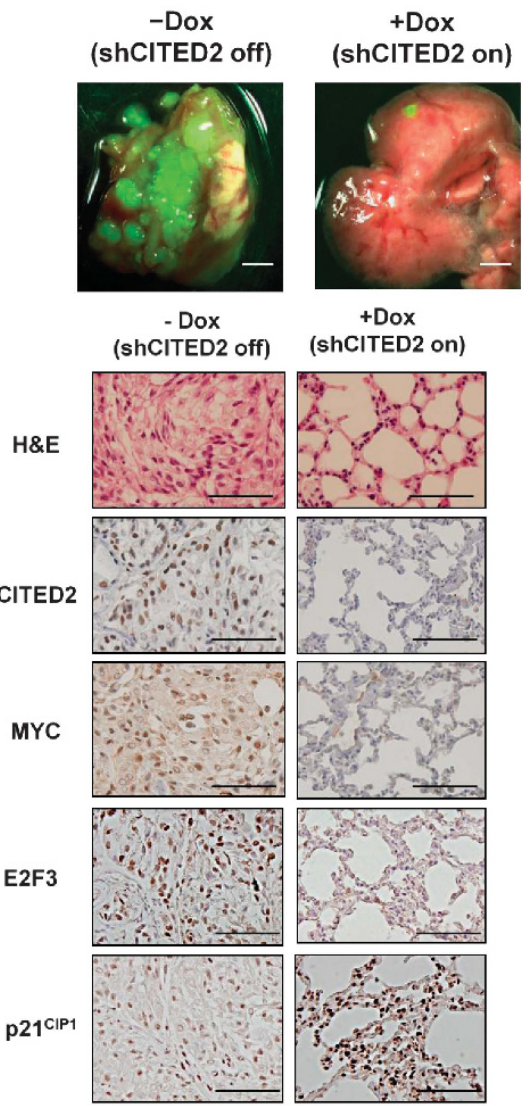

Figure 6 CITED2 enhances lung tumor xenograft growth. (a) Tumor growth of CITED2-overexpressing and vector-only control A549 cells in a subcutaneous xenograft model in nude mice. Tumor volume (top) and tumor weight (bottom left) are shown. The photographs illustrate representative features of tumor growth 8 weeks after injection (bottom right). Error bars indicate the S.E.M. ( $n=10$ mice/group; $\left.{ }^{*} P<0.05\right)$. (b) A549-pLVCT-shCITED2 cells were injected subcutaneously into nude mice. Two weeks after cancer cell injection, mice were treated with or without Dox (200 $\mu \mathrm{g} / \mathrm{ml}$ in drinking water). The photographs illustrate tumor growth 42 days after injection (top). Tumor volume was monitored over time as indicated (bottom). Error bars indicate the S.E.M. ( $n=10$ mice/group; $\left.{ }^{*} P<0.05\right)$. (c) Luciferase/eGFP-labeled A549-pLVCT-shCITED2 cells were orthotopically implanted into the lungs of nude mice. Three days after implantation, mice were treated with or without Dox ( $200 \mu \mathrm{g} / \mathrm{ml})$ in drinking water ( $n=7 \mathrm{mice} / \mathrm{group})$. Tumor growth was monitored based on luciferase activity over time as indicated by IVIS imaging (top), followed by Kaplan-Meier analysis of the overall survival (bottom) of the recipient mice. (d) Excised lungs from the mice in $\mathbf{c}$ were further photographed under bright-field/fluorescence modes (merged image; white scale bars, $0.5 \mathrm{~mm}$; top) and subjected to haematoxylin and eosin ( $\mathrm{H}$ and E) staining combined with immunohistochemistry analysis (bottom). Black scale bars, $25 \mu \mathrm{m}$ 

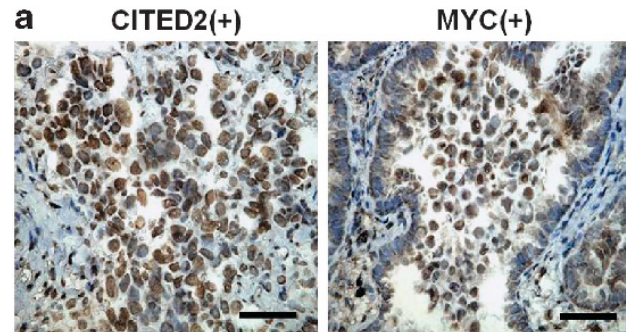

CITED2(-)

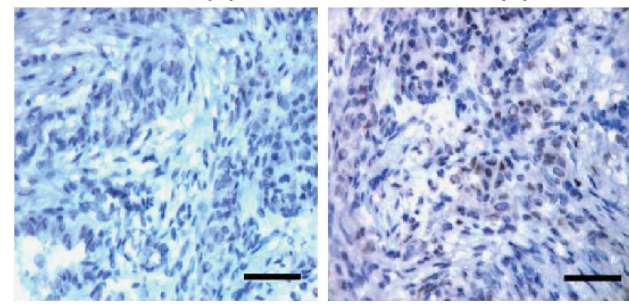

b
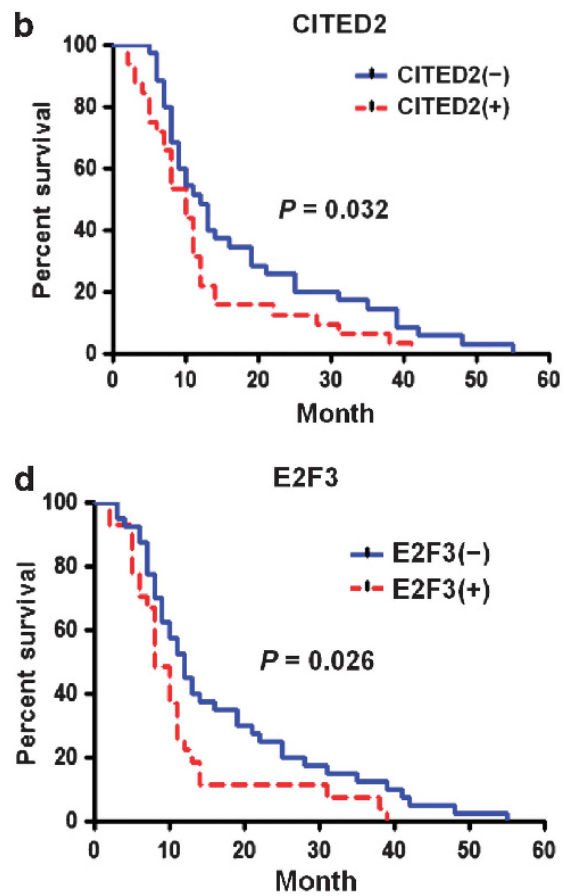

$\mathrm{E} 2 \mathrm{~F} 3(+)$

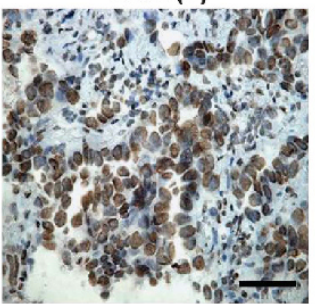

E2F3(-)

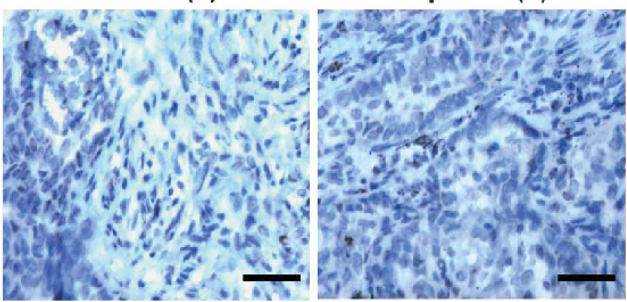

C

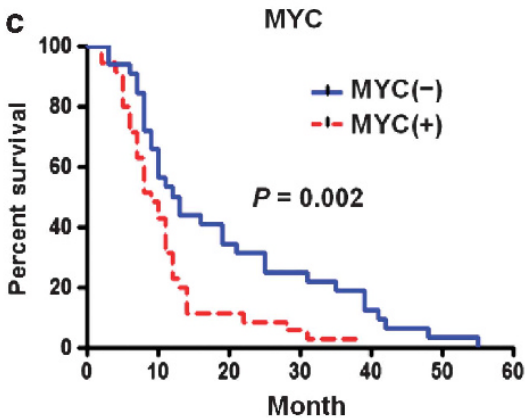

e

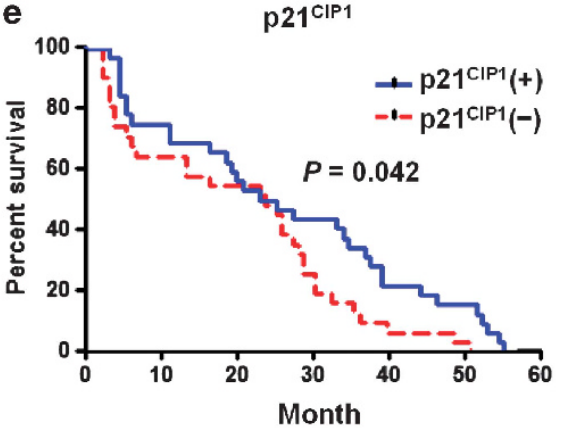

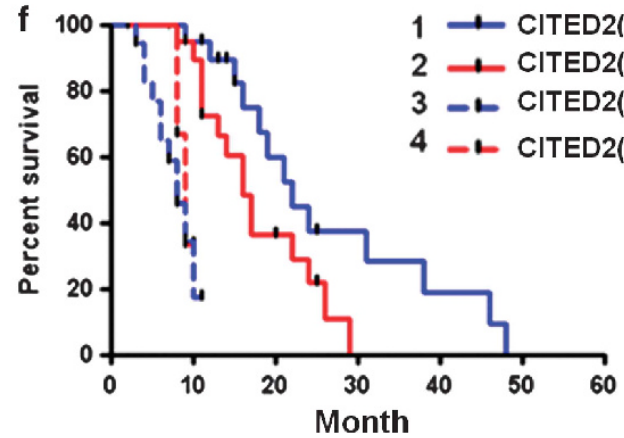

Figure 7 Correlation of CITED2/MYC/E2F3/p21 ${ }^{\mathrm{CIP} 1}$ signaling with the survival rate among patients with NSCLC. (a) Representative results of immunohistochemical staining for CITED2, MYC, E2F3 and p21 ${ }^{\mathrm{CIP} 1}$ in 72 patients with NSCLC. (b-e) Kaplan-Meier analyses of overall survival of patients analyzed in a based on (b) CITED2 expression, (c) MYC expression, (d) E2F3 expression and (e) p21 ClP1 expression. (f) The mean survival times analyzed with different combinations of the four markers CITED2, MYC, E2F3 and p21 ${ }^{\mathrm{CIP} 1}$ were measured by Kaplan-Meier analysis 
triple positive patients, regardless of $\mathrm{p} 21^{\mathrm{ClP} 1}$ status, exhibited a significantly worse prognosis with an exponential decrease in survival as compared with other patients (Figure 7f).

\section{Discussion}

Abnormal cytokine signaling such as that caused by the activating mutation of EGFR or silencing of TGFBRII is often observed in cells from patients with NSCLC. ${ }^{2,5}$ We found that CITED2 responds to TGF- $\alpha / E G F R$ induction and TGF- $\beta$ / TGFBR suppression to orchestrate MYC-mediated proliferation and quiescence, respectively. In addition, CITED2 is induced by many other cytokines. ${ }^{7}$ This cytokine-inducible characteristic of CITED2 and its critical role in cell cycle control lead it to function as a molecular switch for cytokineinduced proliferation and quiescence, and abnormal cytokine signaling could turn on the switch, leading to deregulated growth of neoplastic cells (Figure 8).

It is reported that MYC interacts with p300, and MYC-p300 interaction enhances MYC-mediated transactivation of hTERT promoter. $^{31}$ Consistently, we observed that p300 enhanced MYC-mediated transactivation of E2F3 promoter, and CITED2 expression boosted MYC-p300-mediated transactivation of E2F3 promoter (Figure 3e). We found that MYC bound CITED2 through its C-terminus, and knocking down CITED2 attenuated MYC-p300 interaction. These findings indicate that CITED2 promotes MYC-p300 complex formation and enhances MYC-p300-mediated transactivation of E2F3.

Although it is known that MYC activates E2Fs but represses p21 ${ }^{\mathrm{CIP} 1}$ expression, ${ }^{22,26}$ how MYC can function as both a transcriptional activator and repressor has proven elusive. Here, we showed that CITED2 recruited p300 and HDAC1 to promote MYC-mediated transactivation of E2F3 and transrepression of $p 21^{C I P 1}$. As the binding of MIZ1 and SP1 to the $p 21^{C I P 1}$ promoter are essential for MYC-mediated suppression, ${ }^{21,22}$ whether the MIZ1/SP1/MYC complex predetermines the CITED2-HDAC1 molecules, but not the CITED2-p300 molecules, that are recruited to the p21 CIP1 promoter requires further investigation. The presence of a CITED2-MYC feed-forward network provides an elegant regulatory mechanism to turn on/off or fine-tune a magnitude of MYC-transcriptional activity by modulating CITED2 levels upon cytokine stimulation.

Although we observed that both A549 and H1975 cells responded to TGF- $\beta$-induced cellular quiescence, MYC was downregulated by TGF- $\beta$ in A549 cells but not in H1975 cells. In contrast, CITED2 was downregulated in both A549 and H1975 cells, suggesting that TGF- $\beta$-induced cellular quiescence is mediated by a CITED2-dependent pathway.
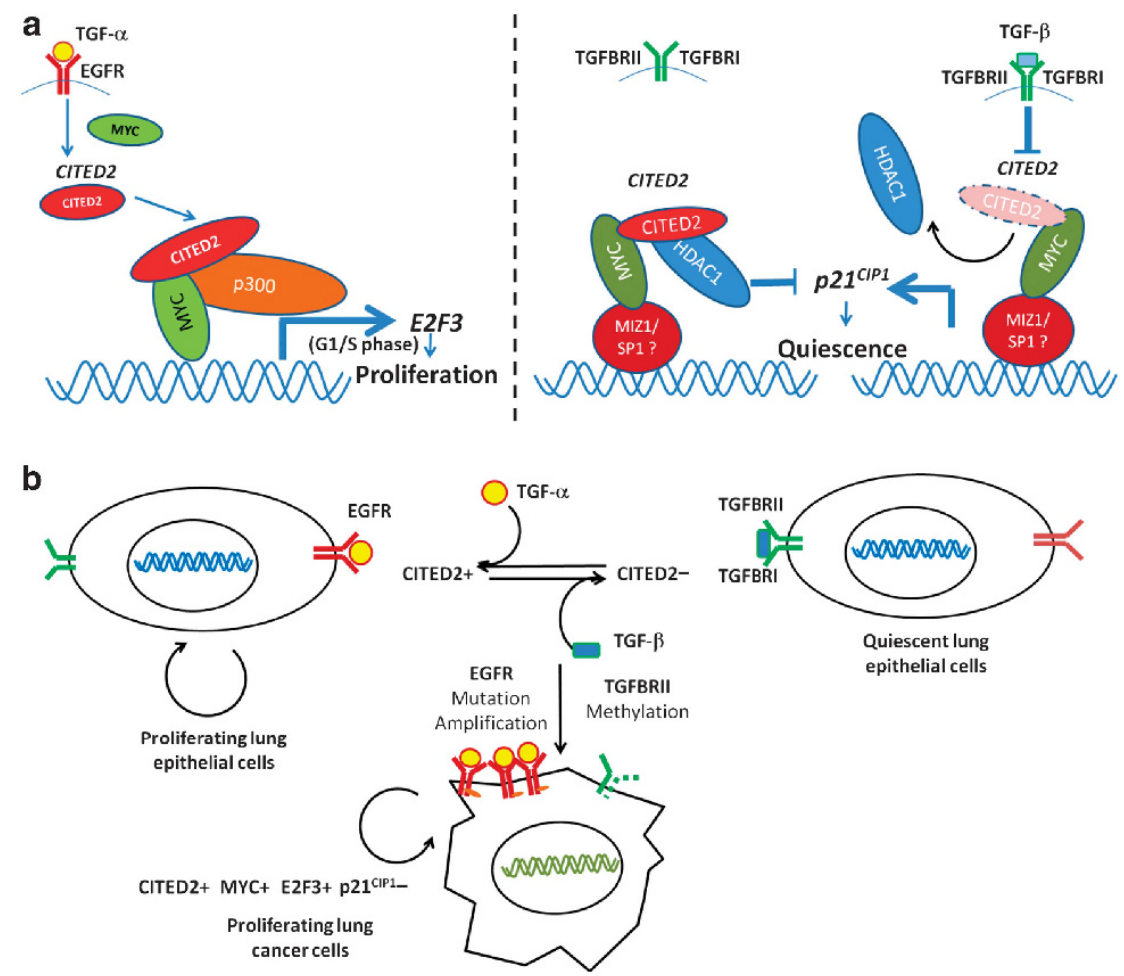

$\mathrm{CITED} 2+\mathrm{MYC}+\mathrm{E} 2 \mathrm{~F} 3+\mathrm{p} 21^{\mathrm{CIP} 1}$

Proliferating lun cancer cells

Figure 8 Cytokine-induced CITED2/MYC/E2F3/p21 ${ }^{\mathrm{CIP} 1}$ signaling in lung cancer. (a) Model of CITED2/MYC/E2F3/p21 ${ }^{\mathrm{CIP} 1}$ signaling. CITED2 expression is induced by TGF- $\alpha / E G F R$ through MYC. Elevated CITED2 levels further recruit p300 to the E2F3 promoter to enhance MYC-mediated transactivation, leading to increased G1/S cell cycle transition (left). TGF- $\beta$ downregulates CITED2, and loss of CITED2 prevents MYC from interacting with HDAC1, causing activation of the $p 21^{1 / P 1}$ promoter and cellular quiescence (right). (b) Model of cytokine-induced homeostasis and neoplastic development of lung epithelial cells. Homeostasis of normal lung epithelial cells is intricately balanced by TGF- $\alpha /$ EGFR proliferation and TGF- $\beta /$ TGFBR quiescence signaling, both of which are fine-tuned by CITED2 expression. Activation of MYC/CITED2/E2F3/ p21 ${ }^{\mathrm{CIP} 1}$ pathway by abnormal cytokine signaling such as EGFR mutation/amplification-activation and TGFBRII silencing contributes to neoplastic development of lung epithelial cells 
Consistent with the previous observation that defective repression of MYC is associated with the loss of TGF- $\beta$ mediated growth arrest, ${ }^{32}$ overexpression of MYC in A549 cells induced CITED2 expression and rendered the cells resistant to TGF- $\beta$-mediated cell growth arrest (Supplementary Figure S6), indicating that the development of TGF- $\beta$ resistance is in part through the induction of CITED2 expression in tumor cells that overexpress MYC. Supporting this notion, knockdown of endogenous CITED2 by Doxinduced shRNA caused cell growth arrest in MYC-overexpressing A549 cells (Supplementary Figure S6). It is indicated that TGF- $\beta$-mediated CITED2 downregulation in MDA-MB-231 cells occurs post-transcriptionally. ${ }^{9}$ Thus, TGF- $\beta$ mediated downregulation of CITED2 is regulated through a MYCdependent transcriptional control and a MYC independent post-transcriptional mechanism (Supplementary Figure S7).

Knockout of CITED2 induces cellular senescence in mouse embryonic fibroblasts through a Bmi-1/CDKN2A-dependent pathway. ${ }^{33}$ Nonetheless, CDKN2A levels were not detected in human lung-cancer cell lines in the presence or absence of shRNA against CITED2; an effect on the expression of Bmi-1 was also not observed after CITED2 knockdown (data not shown). In contrast, p21 ${ }^{\mathrm{CIP} 1}$ did increase in A549 cells after CITED2 knockdown, and $\mathrm{p} 21^{\mathrm{CIP} 1}$ silencing partially rescued the senescence that was induced by CITED2 knockdown, suggesting that $\mathrm{p} 21^{\mathrm{CIP} 1}$ pathway is dominant in lung cancer cells. p2 $1^{\mathrm{CIP} 1}$ blocks the function of E2Fs through direct interaction with E2Fs ${ }^{34}$ or by inhibiting Cyclin/Cdk-mediated phosphorylation of retinoblastoma protein. ${ }^{35}$ The observation that CITED2 modulates both E2F3 and p21 ${ }^{\mathrm{CIP} 1}$ indicates a role for CITED2 in synchronizing E2F3 proliferation and p21 ${ }^{\mathrm{CIP} 1}$ quiescence pathways.

Finally, we observed that molecules in the CITED2/MYC/ E2F3/p2 $1^{\mathrm{CIP} 1}$ pathway are elevated in patients with NSCLC who have a poor prognosis. As MYC, E2F3 and $\mathrm{p} 21^{\mathrm{CIP} 1}$ have been used as prognostic or diagnostic markers in lung cancer, ${ }^{18,36-38}$ our observation that hyperactive CITED2/ $\mathrm{MYC} / \mathrm{E} 2 \mathrm{~F} 3 / \mathrm{p} 21^{\mathrm{CIP} 1}$ signaling is associated with overall survival of patients with NSCLC provides a novel index for predicting disease progression and clinical outcome.

\section{Materials and Methods}

Reagents. Recombinant human TGF- $\alpha$ and TGF- $\beta 1$ were purchased from PeproTech EC (London, UK). Dox (doxycycline hyclate) was ordered from SigmaAldrich (St. Louis, MO, USA).

Cell culture. A549 cells were obtained from the American Type Culture Collection. H1975 cells were kindly provided by Dr. Wayne Chang in the National Health Research Institutes, Taiwan. CL1-0 and CL1-5 cells were established in our laboratory as described. ${ }^{39}$ All lung cancer cell lines tested positive for human origin and were grown in RPMI-1640 medium with 10\% fetal bovine serum.

Plasmid construction. Detailed procedures for construction of CITED2, MYC or EGFR-L858R-expressing lentiviral vectors, CITED2 or E2F3 promoter reporters and shRNA lentiviral vectors can be found in Supplementary Information.

Q-PCR. The Q-PCR assay was performed as described. ${ }^{25}$ Relative gene expression was analyzed using the $2^{-\Delta \Delta C T}$ method using the LightCycler 480 RealTime PCR System (Roche Applied Science, Indianapolis, IN, USA) combined with the Universal Probe Library (Roche Applied Science). Absolute Q-PCR was carried out as described. ${ }^{37} 18 \mathrm{~S}$ rRNA was used as a reference transcript. Primer sequences designed to detect specific genes are listed in Supplementary Table S2.
Luciferase reporter assay. The luciferase assays were performed according to the standard protocols (Dual luciferase reporter assay, Promega, Madison, WI, USA). Renilla luciferase plasmid (pRL-SV40) was co-transfected as an internal control.

Chromatin immunoprecipitation (ChIP). ChIP assay were performed according to the protocol for the LowCell\# ChIP kit (Diagenode, Liege, Belgium) and quantified by Q-PCR. To increase the specificity of Q-PCR in ChIP assay, UPL probe system (Roche Applied Science) was applied in the assay. Antibodies are described in the Supplementary Information. Primer sequences and the corresponding probes designed to detect specific promoters are listed in Supplementary Table S2.

Clonogenic assay. For clonogenic assay with lung cancer cells, 1000 cells/ well in a 6-well plate or 3000 cells/well in a $100 \mathrm{~mm}$ Petri dish were seeded. Colonies were fixed and stained with $3.7 \%$ formaldehyde $/ 80 \%$ methanol $/ 0.25 \%$ crystal violet (Fisher Scientific, Fairlawn, NJ, USA) and counted 2 weeks after plating.

Co-immunoprecipitation assay and western blot analysis. Co-immunoprecipitation assays and western blots were carried out as described. ${ }^{40}$ Antibodies are described in the Supplementary Information.

IF and IHC. IF was performed as previously described. ${ }^{40}$ Antibodies are described in the Supplementary Information.

GST pull-down assay. Flag-tagged CITED2 was transcribed in vitro and translated using TNT Coupled Reticulocyte Lysate Systems (Promega). GST-fused MYC proteins were immortalized on glutathione-Sepharose beads. The GST pull-down assay was performed as described. ${ }^{13}$

Senescence assay. The senescence assay was performed according to the protocol for the Cellular Senescence Detection Kit (Cell Biolabs Inc., San Diego, CA, USA). Cells were washed and transferred to PBS, viewed with an inverted transmission microscope (OLYMPUS IX71, Tokyo, Japan) and documented with a digital imaging system (OLYMPUS C50502, Tokyo, Japan). SA- $\beta$-gal positive cells were quantified by counting positive and negative cells in five random visual fields and averaging the results.

Patients. Patient samples were obtained at the time of surgery in Tri-Service General Hospital and granted by the Institutional Review Board protocol number 099-05-183. All patients gave their informed consent, and the ethics and scientific committees of the participating institutions approved the study. Tumor types were determined according to WHO classification.

Xenograft tumorigenicity assay. Xenograft tumorigenicity was evaluated as described. ${ }^{25}$ Detailed procedures for xenograft tumorigenicity assay and orthotopic implantation can be found in Supplementary Information.

Statistical analysis. Statistical analysis was performed using Student's t-test or a one-way or two-way analysis of variance followed by Turkey's test, as appropriate. $P<0.05$ was considered to be statistically significant. Overall survival rate was assessed using the Kaplan-Meier method and compared using the log-rank test.

\section{Conflict of Interest}

The authors declare no conflict of interest.

Acknowledgements. We thank Dr. Scott Kominsky and Dr. Kristin Webber for their discussion. This research was supported by the Institute of Biomedical Sciences, Academia Sinica, National Yang-Ming University, and the National Science Council (NSC100-2325-B-010-011, NSC100-3112-B-010-004 and NSC100-2321-B-010-021), Executive Yuan, Taiwan, ROC

1. Jetten AM. Growth and differentiation factors in tracheobronchial epithelium. Am J Physiol 1991; 260(6 Pt 1): L361-L373.

2. Sharma SV, Bell DW, Settleman J, Haber DA. Epidermal growth factor receptor mutations in lung cancer. Nat Rev Cancer 2007; 7: 169-181. 
3. Rusch V, Baselga J, Cordon-Cardo C, Orazem J, Zaman M, Hoda S et al. Differential expression of the epidermal growth factor receptor and its ligands in primary non-small cell lung cancers and adjacent benign lung. Cancer Res 1993; 53(10 Suppl): 2379-2385.

4. Antoshina E, Ostrowski LE. TGF beta 1 induces growth arrest and apoptosis but not ciliated cell differentiation in rat tracheal epithelial cell cultures. In Vitro Cell Dev Biol Anim 1997; 33: 212-217.

5. Zhang HT, Chen XF, Wang MH, Wang JC, Qi QY, Zhang RM et al. Defective expression of transforming growth factor beta receptor type II is associated with $\mathrm{CpG}$ methylated promoter in primary non-small cell lung cancer. Clin Cancer Res 2004; 10: 2359-2367.

6. Anumanthan G, Halder SK, Osada H, Takahashi T, Massion PP, Carbone DP et al. Restoration of TGF-beta signalling reduces tumorigenicity in human lung cancer cells. Br J Cancer 2005; 93: 1157-1167.

7. Sun HB, Zhu YX, Yin T, Sledge G, Yang YC. MRG1, the product of a melanocyte-specific gene related gene, is a cytokine-inducible transcription factor with transformation activity. Proc Natl Acad Sci U S A 1998; 95: 13555-13560.

8. Xu B, Qu X, Gu S, Doughman YQ, Watanabe M, Dunwoodie SL et al. Cited2 is required for fetal lung maturation. Dev Biol 2008; 317: 95-105.

9. Chou YT, Yang YC. Post-transcriptional control of Cited2 by transforming growth factor beta. Regulation via Smads and Cited2 coding region. J Biol Chem 2006; 281 : 18451-18462.

10. Glenn DJ, Maurer RA. MRG1 binds to the LIM domain of Lhx2 and may function as a coactivator to stimulate glycoprotein hormone alpha-subunit gene expression. J Biol Chem 1999; 274: 36159-36167.

11. Braganca J, Eloranta JJ, Bamforth SD, Ibbitt JC, Hurst HC, Bhattacharya S. Physical and functional interactions among AP-2 transcription factors, p300/CREB-binding protein, and CITED2. J Biol Chem 2003; 278: 16021-16029.

12. Tien ES, Davis JW, Vanden Heuvel JP. Identification of the CREB-binding protein/p300interacting protein CITED2 as a peroxisome proliferator-activated receptor alpha coregulator. J Biol Chem 2004; 279: 24053-24063.

13. Chou YT, Wang H, Chen Y, Danielpour D, Yang YC. Cited2 modulates TGF-beta-mediated upregulation of MMP9. Oncogene 2006; 25: 5547-5560.

14. Bhattacharya $S$, Michels $C L$, Leung MK, Arany ZP, Kung AL, Livingston DM. Functional role of p35srj, a novel p300/CBP binding protein, during transactivation by HIF-1. Genes Dev 1999; 13: 64-75.

15. Broers JL, Viallet J, Jensen SM, Pass H, Travis WD, Minna JD et al. Expression of c-myc in progenitor cells of the bronchopulmonary epithelium and in a large number of non-small cell lung cancers. Am J Respir Cell Mol Biol 1993; 9: 33-43.

16. Adams MR, Sears R, Nuckolls F, Leone G, Nevins JR. Complex transcriptional regulatory mechanisms control expression of the E2F3 locus. Mol Cell Biol 2000; 20: 3633-3639.

17. Leone $G$, Sears $\mathrm{R}$, Huang $\mathrm{E}$, Rempel R, Nuckolls $\mathrm{F}$, Park $\mathrm{CH}$ et al. Myc requires distinct E2F activities to induce $S$ phase and apoptosis. Mol Cell 2001; 8: 105-113.

18. Cooper CS, Nicholson AG, Foster C, Dodson A, Edwards S, Fletcher A et al. Nuclear overexpression of the E2F3 transcription factor in human lung cancer. Lung Cancer 2006; 54: $155-162$.

19. Reimer D, Hubalek M, Riedle S, Skvortsov S, Erdel M, Concin N et al. E2F3a is critically involved in epidermal growth factor receptor-directed proliferation in ovarian cancer. Cancer Res 2010; 70: 4613-4623.

20. Wu L, Timmers C, Maiti B, Saavedra HI, Sang L, Chong GT et al. The E2F1-3 transcription factors are essential for cellular proliferation. Nature 2001; 414: 457-462.

21. Wu S, Cetinkaya C, Munoz-Alonso MJ, von der Lehr N, Bahram F, Beuger V et al. Myc represses differentiation-induced p21CIP1 expression via Miz-1-dependent interaction with the p21 core promoter. Oncogene 2003; 22: 351-360.
22. Gartel AL, Ye X, Goufman E, Shianov P, Hay N, Najmabadi F et al. Myc represses the p21(WAF1/CIP1) promoter and interacts with Sp1/Sp3. Proc Natl Acad Sci U S A 2001; 98: 4510-4515.

23. Claassen GF, Hann SR. A role for transcriptional repression of p21CIP1 by c-Myc in overcoming transforming growth factor beta -induced cell-cycle arrest. Proc Natl Acad Sci USA 2000; 97: 9498-9503.

24. Nass SJ, Dickson RB. Epidermal growth factor-dependent cell cycle progression is altered in mammary epithelial cells that overexpress c-myc. Clin Cancer Res 1998; 4: 1813-1822.

25. Chou YT, Lin HH, Lien YC, Wang YH, Hong CF, Kao YR et al. EGFR promotes lung tumorigenesis by activating miR-7 through a Ras/ERK/Myc pathway that targets the Ets2 transcriptional repressor ERF. Cancer Res 2010; 70: 8822-8831.

26. Leung JY, Ehmann GL, Giangrande PH, Nevins JR. A role for Myc in facilitating transcription activation by E2F1. Oncogene 2008; 27: 4172-4179.

27. Datto MB, Li Y, Panus JF, Howe DJ, Xiong Y, Wang XF. Transforming growth factor beta induces the cyclin-dependent kinase inhibitor p21 through a p53-independent mechanism. Proc Natl Acad Sci USA 1995; 92: 5545-5549.

28. Katakura Y, Nakata E, Tabira Y, Miura T, Teruya K, Tsuchiya T et al. Decreased tumorigenicity in vivo when transforming growth factor beta treatment causes cancer cell senescence. Biosci Biotechnol Biochem 2003; 67: 815-821.

29. Abbas T, Dutta A. p21 in cancer: intricate networks and multiple activities. Nat Rev Cancer 2009; 9: 400-414.

30. Gui CY, Ngo L, Xu WS, Richon VM, Marks PA. Histone deacetylase (HDAC) inhibitor activation of p21WAF1 involves changes in promoter-associated proteins, including HDAC1. Proc Natl Acad Sci USA 2004; 101: 1241-1246.

31. Faiola F, Liu X, Lo S, Pan S, Zhang K, Lymar E et al. Dual regulation of c-Myc by p300 via acetylation-dependent control of Myc protein turnover and coactivation of Myc-induced transcription. Mol Cell Biol 2005; 25: 10220-10234.

32. Chen CR, Kang Y, Massague J. Defective repression of c-myc in breast cancer cells: a loss at the core of the transforming growth factor beta growth arrest program. Proc Natl Acad Sci USA 2001; 98: 992-999.

33. Kranc KR, Bamforth SD, Braganca J, Norbury C, van Lohuizen M, Bhattacharya S. Transcriptional coactivator Cited2 induces Bmi1 and Mel18 and controls fibroblast proliferation via Ink4a/ARF. Mol Cell Biol 2003; 23: 7658-7666.

34. Afshari CA, Nichols MA, Xiong Y, Mudryj M. A role for a $\mathrm{p} 21-\mathrm{E} 2 \mathrm{~F}$ interaction during senescence arrest of normal human fibroblasts. Cell Growth Differ 1996; 7: 979-988.

35. Harper JW, Adami GR, Wei N, Keyomarsi K, Elledge SJ. The p21 Cdk-interacting protein Cip1 is a potent inhibitor of G1 cyclin-dependent kinases. Cell 1993; 75: 805-816.

36. Hilbe W, Dirnhofer S, Greil R, Woll E. Biomarkers in non-small cell lung cancer prevention. Eur J Cancer Prev 2004; 13: 425-436.

37. Warner KA, Crawford EL, Zaher A, Coombs RJ, Elsamaloty H, Roshong-Denk SL et al. The c-myc x E2F-1/p21 interactive gene expression index augments cytomorphologic diagnosis of lung cancer in fine-needle aspirate specimens. J Mol Diagn 2003; 5: 176-183.

38. Shoji T, Tanaka F, Takata T, Yanagihara K, Otake Y, Hanaoka N et al. Clinical significance of p21 expression in non-small-cell lung cancer. J Clin Oncol 2002; 20: 3865-3871.

39. Chu YW, Yang PC, Yang SC, Shyu YC, Hendrix MJ, Wu R et al. Selection of invasive and metastatic subpopulations from a human lung adenocarcinoma cell line. Am J Respir Cell Mol Biol 1997; 17: 353-360.

40. Hong CF, Chou YT, Lin YS, Wu CW. MAD2B, a novel TCF4-binding protein, modulates TCF4-mediated epithelial-mesenchymal transdifferentiation. $\mathrm{J}$ Biol Chem 2009; $14: 14$. 